\title{
Finite size and geometrical non-linear effects during crack pinning by heterogeneities: An analytical and experimental study
}

\author{
Manish Vasoya ${ }^{\mathrm{a}, \mathrm{b}}$, Aparna Beena Unni ${ }^{\mathrm{a}}$, Jean-Baptiste Leblond ${ }^{\mathrm{a}}$, \\ Veronique Lazarus ${ }^{\mathrm{b}}$, Laurent Ponson ${ }^{\mathrm{a}, *}$ \\ a Sorbonne Universités, UPMC Univ Paris 6 and CNRS, UMR 7190, Institut Jean Le Rond \\ d'Alembert, F-75005 Paris, France \\ ${ }^{\mathrm{b}}$ Univ Paris-Sud and CNRS, UMR 7608, Lab FAST, F-91405, Orsay, France
}

\begin{abstract}
Crack pinning by heterogeneities is a central toughening mechanism in the failure of brittle materials. So far, most analytical explorations of the crack front deformation arising from spatial variations of fracture properties have been restricted to weak toughness contrasts using first order approximation and to defects of small dimensions with respect to the sample size. In this work, we investigate the non-linear effects arising from larger toughness contrasts by extending the approximation to the second order, while taking into account the finite sample thickness. Our calculations predict the evolution of a planar crack lying on the mid-plane of a plate as a function of the material parameters and the loading conditions, especially in the case of a single infinitely elongated obstacle. Peeling experiments are presented which validate the approach and evidence that the second order term broadens its range of validity in terms of toughness contrast values. The work highlights the non-linear response of the crack front to strong defects and the central role played by the thickness of the specimen on the pinning process.
\end{abstract}

Key words: Brittle fracture, crack pinning, perturbation approach, thin film peeling

\section{Introduction}

Predicting the role played by small scale heterogeneities on the macroscopic response of solids is an important challenge in mechanics. In a large range of free-boundary and free-discontinuity problems like wetting of liquids on solid substrates, magnetization of ferromagnetic materials or phase transformations, the relation between microscopic properties and macroscopic behavior is governed by some interface which can be highly sensitive to localized defects. In the context of brittle fracture, this reflects on the strong

* Corresponding author. 
dependence of the toughness to microstructural details, like the spatial distribution of defects and their strength (Gao and Rice, 1989; Bower and Ortiz, 1991; Xia et al., 2012; Patinet et al., 2013b; Démery et al., 2014). The development of predictive tools connecting microstructural parameters of materials to their failure properties is central to engineer materials achieving increased resistance and lifetime. However, to address this challenge, predictive models must consider realistic situations and overcome the limitations of current theories that consider either weak variations of material properties or infinitely small defects compared to the specimen dimensions. The extension of these theories to strong heterogeneities and finite size specimens is the central point of this work.

Microstructural effects on brittle failure phenomena have been largely explored through the fracture mechanics analysis of a continuum elastic medium characterized by some heterogeneous field of local toughness. This approach has met a fair amount of success in capturing experimental observations (Ponson and Bonamy, 2010; Dalmas et al., 2010; Santucci et al., 2010; Xia et al., 2012; Patinet et al., 2013a). In this description, small scale variations of the failure properties locally perturb crack propagation and, in fine, affect the whole failure behavior of the material. The central point in this approach is the prediction of the crack front geometry from the characteristics of local toughness field (spatial distribution of defects, toughness contrast...) and the distribution of the local stress intensity factor along a perturbed crack front (Lazarus, 2011).

Thirty years ago, Rice (1985) derived a first-order formula for the variations of the stress intensity factor induced by some small, but otherwise arbitrary coplanar perturbation of the front of a semi-infinite tensile crack in an infinite body. This expression has been used extensively to predict planar crack growth evolution through random arrays of defects (Schmittbuhl et al., 1995; Ramanathan et al., 1997; Bonamy et al., 2008), and decipher the puzzling geometrical properties of planar cracks observed in experiments (Delaplace et al., 1999; Santucci et al., 2010; Bonamy and Bouchaud, 2011).

However, Rice's first-order formula relies on the assumption that crack front perturbations are of small wavelength compared to the specimen dimensions, which is questionable in some experiments (Schmittbuhl et al., 2003). This was the motivation for Legrand et al. (2011)'s recent extension of Rice (1985)'s formula to the case of coplanar perturbation of an emerging tensile crack lying on the mid-plane of a plate of finite thickness, thus accounting for the effect of the finite dimensions of the specimen. Patinet et al. (2013a) showed that the new formula did significantly improve the agreement between experimental and computed shapes of crack fronts deformed by the presence of obstacles.

Both Rice (1985)'s and Legrand et al. (2011)'s formulae are however accurate only to first order in the perturbation of the front, which limits their application to weak variations of toughness. To explore the non-linear response of cracks pinned by defects of larger contrasts, Leblond et al. (2012) extended Rice (1985)'s first-order formula for a semi-infinite crack in an infinite body to second order, under the assumption of independence of the unperturbed stress intensity factor imposed by the loading with respect to the average crack front location. Then, Vasoya et al. (2013) released this hypothesis, thus extending the range of application of Leblond et al. (2012)'s formula to general loading conditions. Recently, Willis (2013) and Willis and Movchan (2014) investigated the dynamic perturbation of a crack up to second order. Their results, taken in the elastostatic limit for mode 
I loading conditions, were found to be consistent with those previous works.

Still, since both Leblond et al. (2012)'s and Vasoya et al. (2013)'s works considered only infinite bodies, the combined effect of the finite size of the specimens and the geometrical nonlinearities induced by strong defects remains unexplored, and it is the aim of this work to address this question. The results of our calculations apply to several experimental situations involving strong heterogeneities, and for which the crack front perturbation wavelength compares with the thickness of the specimen (Santucci et al., 2010; Chopin et al., 2011). This study also provides predictive tools relevant for the design of heterogeneous materials or thin films where crack pinning by strong engineered obstacles is used to control and modify their failure properties, e.g. to produce asymmetry in the peeling strength of adhesives (Xia et al., 2012).

The paper is organized as follows:

- Section 2 recalls, as a prerequisite, some established results for planar cracks with slightly perturbed fronts. These results pertain to cracks located (i) in some arbitrary body (Rice, 1989), and (ii) on the mid-plane of a plate (Legrand et al., 2011).

- Section 3 presents an extension of Legrand et al. (2011)'s first-order results for a cracked plate to the second order, using Rice (1989)'s general results.

- Section 4 applies these results to the case of a semi-infinite crack propagating quasistatically along the mid-plane of an infinite plate having some heterogeneous distribution of toughness. Assuming the stress intensity factor to be equal to the toughness at every point of the crack front, we determine the resulting shape of this front up to second order in the toughness fluctuations.

- As an application, Section 5 considers the case of a crack penetrating into a single obstacle of infinite length in the direction of propagation. The equilibrium shape of the front is calculated up to second order in the contrast of toughness between the matrix and the obstacle.

- In Section 6, we take inspiration from the experimental setup designed in Xia et al. (2012), and explore the process of front pinning in specimens of thickness comparable to the obstacle size. The geometry of the experimental pinned front is compared with the results of our calculations for various toughness contrasts of the obstacles, providing a critical test of the theory and its range of applicability.

- Section 7 finally provides a summary of, and some comments on, the results obtained.

\section{Summary of some previous works}

\subsection{Rice (1989)'s general formulae for perturbation of a plane crack}

Consider (Figure 1) a planar crack with arbitrary contour embedded in some arbitrary isotropic elastic body symmetric about the crack plane, loaded in pure mode I through some symmetric system of prescribed forces and/or displacements. Assume that the crack front is perturbed, under constant loading, by a small distance $\delta a(s)$ within the crack 
plane in the direction perpendicular to the front, where $s$ denotes a curvilinear abscissa along it.

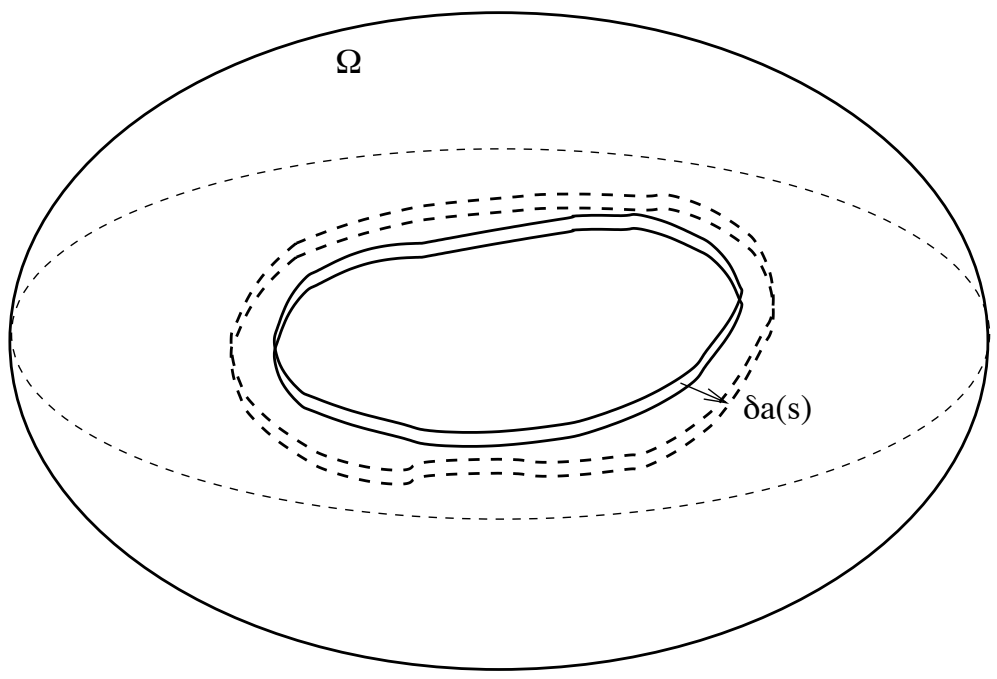

Fig. 1. A planar mode I crack with a slightly perturbed front in an arbitrary body.

Under such conditions, Rice (1989)'s first formula provides the infinitesimal variation $\delta K$ of the local SIF in the following form:

$$
\delta K\left(s_{1}\right)=\left[\delta K\left(s_{1}\right)\right]_{\delta a(s) \equiv \delta a\left(s_{1}\right), \forall s}+P V \int_{C F} Z\left(s_{1}, s\right) K^{0}(s)\left[\delta a(s)-\delta a\left(s_{1}\right)\right] d s .
$$

In this expression,

- $\left[\delta K\left(s_{1}\right)\right]_{\delta a(s) \equiv \delta a\left(s_{1}\right), \forall s}$ denotes the value of $\delta K\left(s_{1}\right)$ for a uniform advance of the front equal to $\delta a\left(s_{1}\right)$;

- the symbol $P V$ denotes the Cauchy principal value of the integral, which is taken over the crack front $C F$;

- $K^{0}(s)$ denotes the local unperturbed SIF at the point $s$ of the front;

- $Z\left(s_{1}, s\right)$ denotes the fundamental kernel $(\mathrm{FK})$ of the cracked geometry considered, tied to Bueckner's mode I crack-face weight function.

The FK depends on the geometry, but has no dependence upon the loading other than on which portions of the body and its boundary have forces versus displacements prescribed. It is not known explicitly except in some special cases, but it is known to obey the following general properties:

$$
\left\{\begin{array}{l}
\text { Symmetry: } Z\left(s_{1}, s_{2}\right)=Z\left(s_{2}, s_{1}\right) . \\
\text { Asymptotic behavior for nearby points: } Z\left(s_{1}, s_{2}\right) \sim \frac{1}{2 \pi\left(s_{1}-s_{2}\right)^{2}} \text { for } s_{1} \rightarrow s_{2} .
\end{array}\right.
$$

Note that the second property shows that the integral in Eq. (1) does make sense as a Cauchy principal value (the function $\delta a$ being assumed to be differentiable).

In addition, Rice (1989)'s second formula provides the infinitesimal variation of the FK 
in the following form:

$$
\delta Z\left(s_{1}, s_{2}\right)=\delta_{*} Z\left(s_{1}, s_{2}\right)+P V \int_{C F} Z\left(s_{1}, s\right) Z\left(s, s_{2}\right)\left[\delta a(s)-\delta_{*} a(s)\right] d s
$$

where $\delta_{*} Z\left(s_{1}, s_{2}\right)$ is the variation of the FK due to any other crack perturbation $\delta_{*} a(s)$ such that $\delta_{*} a\left(s_{1}\right)=\delta a\left(s_{1}\right)$ and $\delta_{*} a\left(s_{2}\right)=\delta a\left(s_{2}\right)$. Note that there are in fact two principal values here, one at $s=s_{1}$ and the other at $s=s_{2}$.

Eq. (3) is interesting, of course, only if special motions $\delta_{*} a(s)$ of the crack front exist for which the variation $\delta_{*} Z\left(s_{1}, s_{2}\right)$ may be calculated independently. It is always possible to find a combination of a translatory motion, a rotation and a homothetical transformation of the crack front satisfying the constraints put on $\delta_{*} a(s)$. In the special case of a semiinfinite crack in an infinite body subjected to prescribed forces only, a combination of a translatory motion and a rotation suffices and leaves the overall geometry unchanged so that $\delta_{*} Z\left(s_{1}, s_{2}\right)$ is zero. In finite bodies, this is no longer the case in general but there are some special cases where certain perturbations $\delta_{*} a(s)$ generate a zero $\delta_{*} Z$. Under such conditions, Eq. (3) reads simply

$$
\delta Z\left(s_{1}, s_{2}\right)=P V \int_{C F} Z\left(s_{1}, s\right) Z\left(s, s_{2}\right)\left[\delta a(s)-\delta_{*} a(s)\right] d s .
$$

\subsection{Legrand et al. (2011)'s solution for a crack lying on the mid-plane of a plate}

Let us now consider (Figure 2a) a semi-infinite plate of thickness $2 h$. Choose the coordinate axes in such a way that this plate occupies the domain $0 \leq x<+\infty,-h \leq y \leq h,-\infty<$ $z<+\infty$ in 3D space. Assume that there is an emerging crack on the mid-plane of the plate, occupying the region $0 \leq x \leq A, y=0,-\infty<z<+\infty$, where $A$, the distance from the plate boundary to the crack front, is supposed to be much larger than $h$. Finally assume that the upper and lower parts of the boundary are subjected to opposite prescribed displacements perpendicular to the crack plane, generating a state of pure mode I at every point of the crack front.

Assume now that the crack front is perturbed by a small distance $\delta a(z)$ within the crack plane $(O z x)$. Under such conditions, Legrand et al. (2011) expressed the infinitesimal variation of the local SIF in the following form:

$$
\frac{\delta K\left(z_{1}\right)}{K^{0}(A)}=-2 \frac{\delta a\left(z_{1}\right)}{A}+P V \int_{-\infty}^{+\infty} \frac{f\left(\frac{A}{h} ; \frac{z-z_{1}}{h}\right)}{\left(z-z_{1}\right)^{2}}\left[\delta a(z)-\delta a\left(z_{1}\right)\right] d z
$$

where $K^{0}(A)$ denotes the unperturbed SIF, depending on the location $A$ of the unperturbed front, and the function $f\left(\frac{A}{h} ; \frac{z-z_{1}}{h}\right) /\left(z_{1}-z\right)^{2}$ represents the $F K$ for the semi-infinite cracked plate considered, subjected to displacements prescribed on its boundary. A fully explicit formula was provided by Legrand et al. (2011) for this specific FK.

An interesting limit-case is that of a semi-infinite crack in an infinite plate $(A \rightarrow+\infty)$. The conditions of prescribed displacements on the boundary then become equivalent, away from it, to conditions of remote prescribed bending moments. In such a limit-case, 
(a)

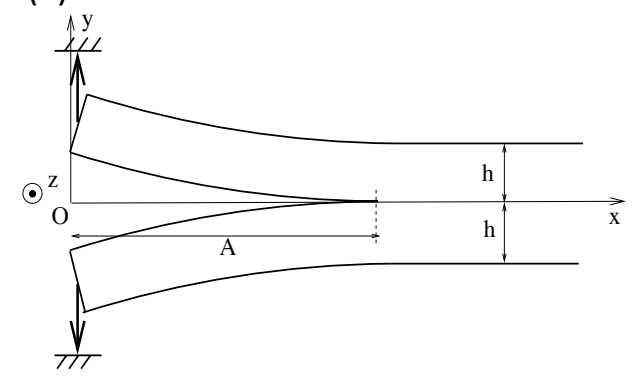

(b)

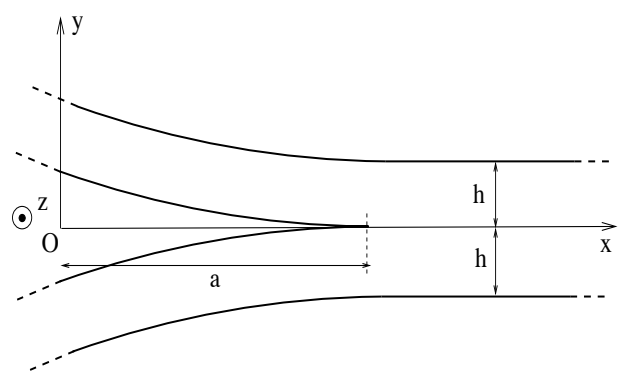

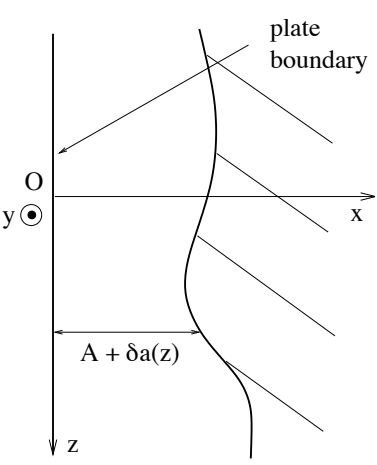

(Crack plane)

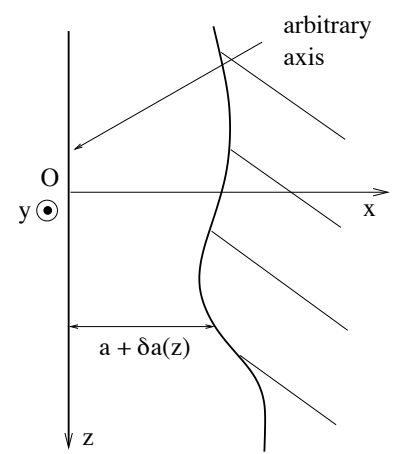

Fig. 2. A slightly perturbed emerging crack lying on the mid-plane of (a) a semi-infinite plate; (b) an infinite plate.

Eq. (5) becomes

$$
\frac{\delta K\left(z_{1}\right)}{K^{0}}=P V \int_{-\infty}^{+\infty} \frac{f\left(\frac{z-z_{1}}{h}\right)}{\left(z-z_{1}\right)^{2}}\left[\delta a(z)-\delta a\left(z_{1}\right)\right] d z
$$

where the function $f\left(\frac{z-z_{1}}{h}\right) /\left(z-z_{1}\right)^{2} \equiv f\left(+\infty ; \frac{z-z_{1}}{h}\right) /\left(z-z_{1}\right)^{2}$ represents the FK for an infinite cracked plate subjected to prescribed forces only. Attention will be focused on this sole special case in the sequel.

It will be necessary to use formulae in Fourier's space. The definition adopted here for the Fourier transform $\widehat{\phi}(k)$ of an arbitrary function $\phi(z)$ is

$$
\phi(z) \equiv \int_{-\infty}^{+\infty} \widehat{\phi}(k) e^{i k z} d k \quad \Leftrightarrow \quad \widehat{\phi}(k) \equiv \frac{1}{2 \pi} \int_{-\infty}^{+\infty} \phi(z) e^{-i k z} d z
$$

The Fourier transform of $\delta K\left(z_{1}\right)$ is readily found by expressing the function $\delta a$ in Eq. (6) in terms of its Fourier transform $\widehat{\delta a}$ : one thus obtains

$$
\frac{\delta K\left(z_{1}\right)}{K^{0}}=\int_{-\infty}^{+\infty} \widehat{\delta a}(k)\left[P V \int_{-\infty}^{+\infty} \frac{f\left(\frac{z-z_{1}}{h}\right)}{\left(z-z_{1}\right)^{2}}\left(e^{i k z}-e^{i k z_{1}}\right) d z\right] d k .
$$

Writing now $e^{i k z}-e^{i k z_{1}}$ as $e^{i k z_{1}}\left(e^{i k\left(z-z_{1}\right)}-1\right)$, one gets from there, with a few transformations,

$$
\frac{\widehat{\delta K}(k)}{K^{0}}=-|k| X(k h) \widehat{\delta a}(k)
$$


where

$$
X(p) \equiv \frac{1}{|p|} P V \int_{-\infty}^{+\infty} \frac{f(u)}{u^{2}}\left(1-e^{i p u}\right) d u \Leftrightarrow f(u) \equiv \frac{1}{2 \pi} \int_{-\infty}^{+\infty}[|p| X(p)]^{\prime \prime} e^{-i p u} d p
$$

Eq. (9) $)_{1}$ here is obtained directly, and Eq. $(9)_{2}$ is then easily established by calculating $[|p| X(p)]^{\prime \prime}$.

In the work of Legrand et al. (2011), Eq. (6) was not obtained directly but through Fourier inversion of Eq. (8). This equation was itself established by solving the elasticity problem of a plate the mid-plane of which contained a crack with a sinusoidal front, both analytically in the case of an infinitesimal plate thickness and by the finite element method in the case of a finite one. The procedure yielded the following explicit expression of the function $X(p)$ :

$$
X(p) \simeq \frac{1}{2}\left[1+\frac{3}{1+\frac{1}{3}|p|^{4 / 3}}\right] .
$$

This expression is (slightly) approximate in general because of the numerical method used to determine it, but exact in the two limits $p \rightarrow 0$ and $p \rightarrow \pm \infty$.

It is finally instructive to consider a sinusoidal perturbation of the front of wavelength $\lambda$. For such a perturbation the only values of the wavenumber $k$ to be considered are $k= \pm 2 \pi / \lambda$. Then:

- if $h \gg \lambda,|k h| \gg 1$ so that, by Eq. (10), $X(k h) \rightarrow \frac{1}{2}$ and Eq. (8) becomes identical to Rice (1985)'s formula for a semi-infinite crack in an infinite body;

- if $h \ll \lambda,|k h| \ll 1$, so that $X(k h) \rightarrow 2$ and Eq. (8) becomes identical to the formula established analytically by Legrand et al. (2011) for a semi-infinite crack lying on the mid-plane of a plate of infinitesimal thickness.

The value of $\delta K(z)$ is thus 4 times larger, for a given crack front perturbation $\delta a(z)$, in a very thin plate than in an infinite body.

\section{Coplanar perturbation of a semi-infinite crack lying on the mid-plane of an infinite plate}

\subsection{Notations}

For now on, we consider (Figure $2 \mathrm{~b}$ ) the case of a semi-infinite crack located on the midplane of an infinite plate of thickness $2 h$, loaded in pure mode I through prescribed forces only.

Assume that the crack front perturbation $\delta a(z)$ is of the form

$$
\delta a(z)=\epsilon \phi(z)
$$


where $\epsilon$ is a small parameter and $\phi(z)$ a given, fixed, smooth function. With this notation the equation of the perturbed front reads

$$
x(z)=a+\epsilon \phi(z)
$$

where $a$ denotes the distance from some arbitrary reference axis $O z$ to the unperturbed straight front. The position of the perturbed front is thus characterized by the parameters $a$ and $\epsilon$, and the position of a current point along it by the parameter $z$.

\subsection{First-order expansion of the fundamental kernel}

The FK for the crack configuration depicted by Eq.(12) is denoted $Z\left(\epsilon ; z_{1}, z_{2}\right)$, and our interest here lies in the first-order expression of $Z\left(\epsilon ; z_{1}, z_{2}\right)$,

$$
Z\left(\epsilon ; z_{1}, z_{2}\right) \equiv Z^{0}\left(z_{1}, z_{2}\right)+\epsilon Z^{1}\left(z_{1}, z_{2}\right)+O\left(\epsilon^{2}\right) .
$$

Since both the special loading considered by Legrand et al. (2011) when deriving Eqs. (6), (9) and (10), and the more general one considered here, involve prescribed forces only (no prescribed displacements), the $\mathrm{FK} Z^{0}\left(z_{1}, z_{2}\right)$ for the unperturbed configuration of the crack is the same as in Eq. (6),

$$
Z^{0}\left(z_{1}, z_{2}\right) \equiv \frac{f\left(\frac{z_{1}-z_{2}}{h}\right)}{\left(z_{1}-z_{2}\right)^{2}}
$$

where the function $f$ is given by Eqs. (9) and (10).

In the specific case considered, the ancillary perturbation $\delta_{*} a(z) \equiv \epsilon \phi_{*}(z)$ of Rice's second formula (3) may be taken as a suitable combination of a translatory motion and a rotation:

$$
\phi_{*}(z) \equiv \phi\left(z_{1}\right)+\frac{\phi\left(z_{2}\right)-\phi\left(z_{1}\right)}{z_{2}-z_{1}}\left(z-z_{1}\right)=\phi\left(z_{2}\right)+\frac{\phi\left(z_{1}\right)-\phi\left(z_{2}\right)}{z_{1}-z_{2}}\left(z-z_{2}\right) .
$$

The lack of a homothetical transformation of the crack front here (which basically arises from its initial straightness) implies that it undergoes a mere rigid-body motion. Under such a motion the thickness of the plate, and therefore the overall geometry, remain unchanged ${ }^{1}$ so that $\delta_{*} Z$ is zero. Eq. (4) therefore applies and gives

$$
Z^{1}\left(z_{1}, z_{2}\right)=P V \int_{-\infty}^{+\infty} \frac{f\left(\frac{z_{1}-z}{h}\right) f\left(\frac{z-z_{2}}{h}\right)}{\left(z_{1}-z\right)^{2}\left(z-z_{2}\right)^{2}}\left[\phi(z)-\phi_{*}(z)\right] d z
$$

where use has been made of Eq. (14).

$\overline{1}$ Note that the hypothesis of infiniteness of the plate plays a central role here; if it were semiinfinite, like at the beginning of Section 2.2, the rotation of the crack front would not leave the geometry unchanged since this front would not remain parallel to the plate boundary. 


\subsection{Second-order expansion of the stress intensity factor in the physical space}

The mode I SIF for a given, fixed loading imposed upon the cracked geometry is denoted $K(a, \epsilon ; z)$. Our interest here lies in the second-order expression of this SIF with respect to $\epsilon$ :

$$
K(a, \epsilon ; z) \equiv K^{0}(a)+\epsilon K^{1}(a ; z)+\epsilon^{2} K^{2}(a ; z)+O\left(\epsilon^{3}\right) .
$$

It is assumed in this equation that the loading has a translatory invariance in the direction $z$ of the crack front, so that the unperturbed SIF $K^{0}(a)$ depends on the location $a$ of the (straight) front but not on the position of the point of observation along it.

At order 1 , the expression of $K^{1}(a ; z)$ is obtained through direct application of Rice (1989)'s first formula (1) for the variation of the SIF to the straight configuration of the front:

$$
K^{1}\left(a ; z_{1}\right)=\frac{d K^{0}}{d a}(a) \phi\left(z_{1}\right)+K^{0}(a) P V \int_{-\infty}^{+\infty} \frac{f\left(\frac{z-z_{1}}{h}\right)}{\left(z-z_{1}\right)^{2}}\left[\phi(z)-\phi\left(z_{1}\right)\right] d z
$$

This equation is identical to Eq. (6) of Legrand et al. (2011) except for the additional first term in the right-hand side. This term was zero for the special loading envisaged by Legrand et al. (2011) because the unperturbed SIF was independent of the location of the (straight) crack front within the crack plane, but this does not remain true for the more general loading considered here.

In the same way, at order $2, K^{2}(a ; z)$ may be obtained by again applying Rice (1989)'s first formula (1), considering some pre-perturbed configuration of the front upon which is superimposed a secondary, infinitesimal proportional perturbation. When doing so, one must use formulae for the SIF and the FK on the pre-perturbed configuration accurate to first order in the primary perturbation; the first of these formulae is provided by Eq. (18) and the second by Rice (1989)'s second formula (3), which takes the form (16) in the present case. The output is an expression of $\partial K(a, \epsilon ; z) / \partial \epsilon$ accurate to first order in $\epsilon$, which immediately yields the second-order expression of $K(a, \epsilon ; z)$ upon integration.

This was the procedure followed by Leblond et al. (2012) and Vasoya et al. (2013) in the case of an infinite body. The reasoning for a plate is analogous and therefore will not be repeated. The final result reads:

$$
\begin{aligned}
K^{2}\left(a ; z_{1}\right)= & \frac{1}{2} \frac{d^{2} K^{0}}{d a^{2}}(a)\left[\phi\left(z_{1}\right)\right]^{2}+\frac{1}{2} \frac{d K^{0}}{d a}(a) P V \int_{-\infty}^{+\infty} \frac{f\left(\frac{z-z_{1}}{h}\right)}{\left(z-z_{1}\right)^{2}}\left[\phi^{2}(z)-\phi^{2}\left(z_{1}\right)\right] d z \\
& +\frac{K^{0}(a)}{2}\left\{P V \int_{-\infty}^{+\infty} \int_{-\infty}^{+\infty} \frac{f\left(\frac{z-z_{1}}{h}\right) f\left(\frac{z^{\prime}-z}{h}\right)}{\left(z-z_{1}\right)^{2}\left(z^{\prime}-z\right)^{2}}\left[\phi(z)-\phi\left(z_{1}\right)\right]\left[\phi\left(z^{\prime}\right)-\phi(z)\right] d z d z^{\prime}\right. \\
& +P V \int_{-\infty}^{+\infty} \int_{-\infty}^{+\infty} \frac{f\left(\frac{z^{\prime}-z_{1}}{h}\right) f\left(\frac{z^{\prime}-z}{h}\right)}{\left(z^{\prime}-z_{1}\right)^{2}\left(z^{\prime}-z\right)^{2}}\left[\phi\left(z^{\prime}\right)-\phi\left(z_{1}\right)-\frac{\phi(z)-\phi\left(z_{1}\right)}{z-z_{1}}\left(z^{\prime}-z_{1}\right)\right] \\
& \left.\times\left[\phi(z)-\phi\left(z_{1}\right)\right] d z d z^{\prime}\right\}
\end{aligned}
$$


3.4 Second-order expansions of the stress intensity factor and the energy-release-rate in Fourier's space

With regard to the SIF, at order 1 , the expression of the Fourier transform of $K^{1}\left(a ; z_{1}\right)$ is easily established by expressing the function $\phi$ in Eq. (18) in terms of its Fourier transform $\widehat{\phi}$ and using Eq. $(9)_{1}$; one thus gets

$$
\widehat{K^{1}}(a ; k)=\left[\frac{d K^{0}}{d a}(a)-K^{0}(a)|k| X(k h)\right] \widehat{\phi}(k) .
$$

This expression extends Eq. (8), due to Legrand et al. (2011), by including the term $\frac{d K^{0}}{d a}(a) \widehat{\phi}(k)$, which was absent in this equation since for the loading considered by these authors, the unperturbed SIF $K^{0}$ was independent of the location of the crack front within the crack plane.

In the same way, at order 2 , the expression of the Fourier transform of $K^{2}\left(a ; z_{1}\right)$ can be established by expressing the function $\phi$ in Eq. (19) in terms of its Fourier transform $\widehat{\phi}$; one thus gets

$$
\begin{aligned}
K^{2}\left(a ; z_{1}\right)= & \int_{-\infty}^{+\infty} \int_{-\infty}^{+\infty} \widehat{\phi}(k) \widehat{\phi}\left(k^{\prime}\right)\left\{\frac{1}{2} \frac{d^{2} K^{0}}{d a^{2}}(a) e^{i\left(k+k^{\prime}\right) z_{1}}\right. \\
& +\frac{1}{2} \frac{d K^{0}}{d a}(a) P V \int_{-\infty}^{+\infty} \frac{f\left(\frac{z-z_{1}}{h}\right)}{\left(z-z_{1}\right)^{2}}\left(e^{i\left(k+k^{\prime}\right) z}-e^{i\left(k+k^{\prime}\right) z_{1}}\right) d z \\
& +\frac{K^{0}(a)}{2}\left[P V \int_{-\infty}^{+\infty} \int_{-\infty}^{+\infty} \frac{f\left(\frac{z-z_{1}}{h}\right) f\left(\frac{z^{\prime}-z}{h}\right)}{\left(z-z_{1}\right)^{2}\left(z^{\prime}-z\right)^{2}}\left(e^{i k z}-e^{i k z_{1}}\right)\left(e^{i k^{\prime} z^{\prime}}-e^{i k^{\prime} z}\right) d z d z^{\prime}\right. \\
& +P V \int_{-\infty}^{+\infty} \int_{-\infty}^{+\infty} \frac{f\left(\frac{z^{\prime}-z_{1}}{h}\right) f\left(\frac{z^{\prime}-z}{h}\right)}{\left(z^{\prime}-z_{1}\right)^{2}\left(z^{\prime}-z\right)^{2}}\left(e^{i k z^{\prime}}-e^{i k z_{1}}-\frac{e^{i k z}-e^{i k z_{1}}}{z-z_{1}}\left(z^{\prime}-z_{1}\right)\right) \\
& \left.\left.\times\left(e^{i k^{\prime} z}-e^{i k^{\prime} z_{1}}\right) d z d z^{\prime}\right]\right\} d k d k^{\prime}
\end{aligned}
$$

Now, taking $e^{i\left(k+k^{\prime}\right) z_{1}}$ as a common factor in the integrands and using Eq. $(9)_{1}$, one gets

$$
\begin{aligned}
K^{2}\left(a ; z_{1}\right)= & \int_{-\infty}^{+\infty} \int_{-\infty}^{+\infty} \widehat{\phi}(k) \widehat{\phi}\left(k^{\prime}\right)\left\{\frac{1}{2} \frac{d^{2} K^{0}}{d a^{2}}(a)-\frac{1}{2} \frac{d K^{0}}{d a}(a)\left|k+k^{\prime}\right| X\left(\left(k+k^{\prime}\right) h\right)\right. \\
& \left.+\frac{K^{0}(a)}{2}\left[I_{1}\left(h ; k, k^{\prime} ; z_{1}\right)+I_{2}\left(h ; k, k^{\prime} ; z_{1}\right)\right]\right\} e^{i\left(k+k^{\prime}\right) z_{1}} d k d k^{\prime}
\end{aligned}
$$

where $I_{1}\left(h ; k, k^{\prime} ; z_{1}\right)$ and $I_{2}\left(h ; k, k^{\prime} ; z_{1}\right)$ are double integrals, the expression and calculation of which is presented in Appendix A, with the following results:

$$
\left\{\begin{aligned}
I_{1}\left(h ; k, k^{\prime} ; z_{1}\right) \equiv I_{1}\left(h ; k, k^{\prime}\right)= & \left|k^{\prime}\right| X\left(k^{\prime} h\right)\left[\left|k+k^{\prime}\right| X\left(\left(k+k^{\prime}\right) h\right)-\left|k^{\prime}\right| X\left(k^{\prime} h\right)\right] \\
I_{2}\left(h ; k, k^{\prime} ; z_{1}\right) \equiv I_{2}\left(h ; k, k^{\prime}\right)= & \frac{1}{2}\left[k^{2} X^{2}(k h)-\left(k+k^{\prime}\right)^{2} X^{2}\left(\left(k+k^{\prime}\right) h\right)-k^{\prime 2} X^{2}\left(k^{\prime} h\right)\right. \\
& \left.+2\left|k+k^{\prime}\right|\left|k^{\prime}\right| X\left(\left(k+k^{\prime}\right) h\right) X\left(k^{\prime} h\right)\right] .
\end{aligned}\right.
$$

Equation (22) becomes, upon use of these formulae and "symmetrization" of the integrand 
with respect to $k$ and $k^{\prime}$,

$$
K^{2}\left(a ; z_{1}\right)=K^{0}(a) \int_{-\infty}^{+\infty} \int_{-\infty}^{+\infty} R\left(a ; h ; k, k^{\prime}\right) \widehat{\phi}(k) \widehat{\phi}\left(k^{\prime}\right) e^{i\left(k+k^{\prime}\right) z_{1}} d k d k^{\prime}
$$

where

$$
\begin{aligned}
R\left(a ; h ; k, k^{\prime}\right) \equiv & \frac{1}{2} \frac{d^{2} K^{0}}{K^{0} d a^{2}}(a)-\frac{1}{2} \frac{d K^{0}}{K^{0} d a}(a)\left|k+k^{\prime}\right| X\left(\left(k+k^{\prime}\right) h\right) \\
& +\frac{\left|k+k^{\prime}\right|}{2} X\left(\left(k+k^{\prime}\right) h\right)\left[|k| X(k h)+\left|k^{\prime}\right| X\left(k^{\prime} h\right)\right] \\
& -\frac{1}{4}\left[\left(k+k^{\prime}\right)^{2} X^{2}\left(\left(k+k^{\prime}\right) h\right)+k^{2} X^{2}(k h)+k^{\prime 2} X^{2}\left(k^{\prime} h\right)\right] .
\end{aligned}
$$

Using the change of variable $k_{1}=k+k^{\prime}$, the preceding expression of $K^{2}\left(a ; z_{1}\right)$ may be rewritten in the form

$$
K^{2}\left(a ; z_{1}\right)=K^{0}(a) \int_{-\infty}^{+\infty}\left[\int_{-\infty}^{+\infty} R\left(a ; h ; k, k_{1}-k\right) \widehat{\phi}(k) \widehat{\phi}\left(k_{1}-k\right) d k\right] e^{i k_{1} z_{1}} d k_{1}
$$

which implies, upon comparison with the definition (7) of the Fourier transform, that

$$
\widehat{K^{2}}\left(a ; k_{1}\right)=K^{0}(a) \int_{-\infty}^{+\infty} R\left(a ; h ; k, k_{1}-k\right) \widehat{\phi}(k) \widehat{\phi}\left(k_{1}-k\right) d k .
$$

Note the remarkable property that $\widehat{K^{2}}\left(a ; k_{1}\right)$, just like $\widehat{K^{1}}\left(a ; k_{1}\right)$, depends upon the FK of the cracked geometry considered through the sole function $X$ defined by Eq. (9) (since the expression (25) of $R\left(a ; h ; k, k^{\prime}\right)$ involves only $\left.X\right)$.

A comparison with the results of Vasoya et al. (2013) for an infinite body is in order here, by letting $h$ go to infinity. The function $R$ becomes in this limit, since $X(k h) \rightarrow \frac{1}{2}$ (see Eq. (10)):

$$
\begin{aligned}
\lim _{h \rightarrow+\infty} R\left(a ; h ; k, k^{\prime}\right)= & \frac{1}{2} \frac{d^{2} K^{0}}{K^{0} d a^{2}}(a)-\frac{1}{4} \frac{d K^{0}}{K^{0} d a}(a)\left|k+k^{\prime}\right| \\
& +\frac{1}{16}\left[2\left|k+k^{\prime}\right|\left(|k|+\left|k^{\prime}\right|\right)-\left(k+k^{\prime}\right)^{2}-k^{2}-k^{\prime 2}\right]
\end{aligned}
$$

which is another form of the function $R$ found by Vasoya et al. (2013) (and noted $P$ in their paper). This establishes the consistency of the results with those found in the case of an infinite body. Interestingly, Eq. (27) is also consistent with the calculations up to second order of Willis (2013) who considered the more general situation of the dynamic in-plane perturbation of a mode I crack. Indeed, specializing his results to some sinusoidal front perturbation $\phi(z)=\epsilon \cos (k z)$ in the elastostatic limit, his calculations lead to $K^{2}=-\frac{1}{8} K^{0}(k \epsilon)^{2} \sin ^{2}(k z)$ in agreement with our results for loading conditions invariant in the propagation direction, i.e. such that $\frac{d K^{0}}{d a}=0$.

The expansion of the Fourier transform $\widehat{G}(a, \epsilon ; k)$ of the elastic energy-release-rate $G(a, \epsilon ; z)$ follows from Irwin's formula and the expressions $(20)$ and $(26)$ of $\widehat{K^{1}}(a ; k)$ and $\widehat{K^{2}}(a ; k)$ :

$$
\widehat{G}(a, \epsilon ; k) \equiv G^{0}(a) \delta(k)+\epsilon \widehat{G^{1}}(a ; k)+\epsilon^{2} \widehat{G^{2}}(a ; k)+O\left(\epsilon^{3}\right)
$$


where $\delta$ denotes Dirac's function, $G^{0}(a)$ the unperturbed elastic energy-release-rate, and

$$
\left\{\begin{array}{l}
\widehat{G^{1}}(a ; k)=\left[\frac{d G^{0}}{d a}(a)-2 G^{0}(a)|k| X(k h)\right] \widehat{\phi}(k) \\
\widehat{G^{2}}\left(a ; k_{1}\right)=G^{0}(a) \int_{-\infty}^{+\infty} S\left(a ; h ; k, k_{1}-k\right) \widehat{\phi}(k) \widehat{\phi}\left(k_{1}-k\right) d k
\end{array}\right.
$$

where

$$
\begin{aligned}
S\left(a ; h ; k, k^{\prime}\right) \equiv & \frac{1}{2} \frac{d^{2} G^{0}}{G^{0} d a^{2}}(a)-\frac{1}{2} \frac{d G^{0}}{G^{0} d a}(a)\left[\left|k+k^{\prime}\right| X\left(\left(k+k^{\prime}\right) h\right)+|k| X(k h)+\left|k^{\prime}\right| X\left(k^{\prime} h\right)\right] \\
& -\frac{1}{2}\left[\left|k+k^{\prime}\right| X\left(\left(k+k^{\prime}\right) h\right)-|k| X(k h)-\left|k^{\prime}\right| X\left(k^{\prime} h\right)\right]^{2} \\
& +2|k|\left|k^{\prime}\right| X(k h) X\left(k^{\prime} h\right) .
\end{aligned}
$$

\section{The equilibrium shape of the front of a crack propagating in a heteroge- neous plate}

We shall now apply the preceding results to the study of coplanar propagation of a crack along the mid-plane of a plate, governed by Griffith's criterion with a heterogeneous fracture toughness $G_{c}(x, z)$ given by

$$
G_{c}(x, z) \equiv \overline{G_{c}}\left[1+\epsilon g_{c}(x, z)\right]
$$

where $\overline{G_{c}}$ is a "mean toughness", $\epsilon$ a small parameter and $g_{c}(x, z)$ a given dimensionless function describing the toughness fluctuations. For a given loading, provided that $G$ is equal to $G_{c}$ at every point of the crack front, the distribution of toughness determines the shape of this front in the form

$$
x=a+\epsilon \phi^{1}(a ; z)+\epsilon^{2} \phi^{2}(a ; z)+O\left(\epsilon^{3}\right)
$$

where $a, \phi^{1}(a ; z)$ and $\phi^{2}(a ; z)$ are a parameter and some functions to be determined.

For the crack front shape depicted by Eq. (32), corresponding to the perturbation function $\delta a \equiv \epsilon \phi^{1}+\epsilon^{2} \phi^{2}$, the expression (28) of $\widehat{G}$ takes the form

$$
\begin{aligned}
\widehat{G}(a, \epsilon ; k)= & G^{0}(a) \delta(k)+\epsilon \widehat{\mathcal{G}^{1}}\left[a ;\left\{\widehat{\phi^{1}}\right\}\right](k) \\
& +\epsilon^{2}\left\{\widehat{\mathcal{G}^{1}}\left[a ;\left\{\widehat{\phi^{2}}\right\}\right](k)+\widehat{\mathcal{G}^{2}}\left[a ;\left\{\widehat{\phi^{1}}\right\}\right](k)\right\}+O\left(\epsilon^{3}\right)
\end{aligned}
$$

where $\widehat{\mathcal{G}^{1}}[a ;\{\widehat{\phi}\}]$ and $\widehat{\mathcal{G}^{2}}[a ;\{\widehat{\phi}\}]$ are the linear and quadratic functionals of the function $\widehat{\phi}$ defined by Eqs. $(29)_{1}$ and $(29)_{2}$ respectively. Also, the value of the local toughness along the crack front is

$$
\begin{aligned}
& G_{c}\left[x=a+\epsilon \phi^{1}(a ; z)+\epsilon^{2} \phi^{2}(a ; z)+O\left(\epsilon^{3}\right), z\right] \\
& =\overline{G_{c}}\left[1+\epsilon g_{c}(a, z)+\epsilon^{2} \frac{\partial g_{c}}{\partial x}(a, z) \phi^{1}(a ; z)\right]+O\left(\epsilon^{3}\right) .
\end{aligned}
$$


The Fourier transform of this expression at the point $k_{1}$ is

$$
\overline{G_{c}}\left[\delta\left(k_{1}\right)+\epsilon \widehat{g}_{c}\left(a, k_{1}\right)+\epsilon^{2} \int_{-\infty}^{+\infty} \frac{\partial \widehat{g}_{c}}{\partial x}(a, k) \widehat{\phi^{1}}\left(a ; k_{1}-k\right) d k\right]+O\left(\epsilon^{3}\right) .
$$

Equating the right-hand side of equation (33) (at $k=k_{1}$ ) to this expression, one gets the following conditions:

- At order 0:

$$
G^{0}(a)=\overline{G_{c}}
$$

This condition determines the mean location $a$ of the crack front.

- At order 1:

$$
\widehat{\mathcal{G}^{1}}\left[a ;\left\{\widehat{\phi^{1}}\right\}\right](k)=\overline{G_{c}} \widehat{g}_{c}(a, k),
$$

which implies, by the expression $(29)_{1}$ of the functional $\widehat{\mathcal{G}^{1}}$ and equation (34), that

$$
\widehat{\phi^{1}}(a ; k)=-\frac{\widehat{g_{c}}(a, k)}{2|k| X(k h)-\frac{d G^{0}}{G^{0} d a}(a)} .
$$

- At order 2:

$$
\widehat{\mathcal{G}^{1}}\left[a ;\left\{\widehat{\phi^{2}}\right\}\right]\left(k_{1}\right)=-\widehat{\mathcal{G}^{2}}\left[a ;\left\{\widehat{\phi^{1}}\right\}\right]\left(k_{1}\right)+\overline{G_{c}} \int_{-\infty}^{+\infty} \frac{\partial \widehat{g_{c}}}{\partial x}(a, k) \widehat{\phi^{1}}\left(a ; k_{1}-k\right) d k,
$$

which implies, by equations (29), (34) and (35), that

$$
\begin{aligned}
\widehat{\phi^{2}}\left(a ; k_{1}\right)= & \frac{1}{2\left|k_{1}\right| X\left(k_{1} h\right)-\frac{d G^{0}}{G^{0} d a}(a)}\left\{\int_{-\infty}^{+\infty} S\left(a ; h ; k, k_{1}-k\right)\right. \\
& \times \frac{\widehat{g_{c}}(a, k)}{2|k| X(k h)-\frac{d G^{0}}{G^{0} d a}(a)} \frac{\widehat{g_{c}}\left(a, k_{1}-k\right)}{2\left|k_{1}-k\right| X\left(\left(k_{1}-k\right) h\right)-\frac{d G^{0}}{G^{0} d a}(a)} d k \\
& \left.+\int_{-\infty}^{+\infty} \frac{\partial \widehat{g_{c}}}{\partial x}(a, k) \frac{\widehat{g_{c}}\left(a, k_{1}-k\right)}{2\left|k_{1}-k\right| X\left(\left(k_{1}-k\right) h\right)-\frac{d G^{0}}{G^{0} d a}(a)} d k\right\} .
\end{aligned}
$$

As discussed in Vasoya et al. (2013), the convergence of the integrals involved in the expressions (35) and $(36)$ of $\widehat{\phi^{1}}(a ; k)$ and $\widehat{\phi^{2}}(a ; k)$ and their inverse Fourier transforms requires

$$
\frac{d G^{0}}{d a}(a)<0
$$

This condition indeed ensures that the denominators of the various fractions never vanish since $X(p)>0$. This hypothesis is natural since it warrants stability of crack propagation in the case of a homogeneous toughness (straight crack front). It was satisfied in all experiments mentioned in the Introduction, and will also be so in those discussed in the sequel. 


\section{The shape of a crack front encountering a single obstacle}

\subsection{Toughness map}

As an application, we shall determine the equilibrium shape of the front of a crack lying on the mid-plane of an infinite plate and penetrating into a single obstacle of infinite length in the direction of propagation, up to second order in the contrast of toughness. The toughness of the matrix will be denoted $G_{c}^{\mathrm{M}}$, and the toughness and width of the obstacle, $G_{c}^{\mathrm{O}}$ and $2 d$, respectively (see Fig. 3). We shall be particularly interested in the limit-case where $\frac{d G^{0}}{d a}(a) \rightarrow 0^{-}$, corresponding to the situation where the typical distance of variation of the unperturbed elastic energy-release-rate is much larger than $d$.

The toughness distribution may be represented by formula (31) with

$$
\overline{G_{c}} \equiv G_{c}^{\mathrm{M}} \quad ; \quad \epsilon \equiv \frac{G_{c}^{\mathrm{O}}-G_{c}^{\mathrm{M}}}{G_{c}^{\mathrm{M}}} \quad ; \quad g_{c}(x, z) \equiv g_{c}(z) \equiv \begin{cases}1 & \text { if }|z|<d \\ 0 & \text { if }|z|>d\end{cases}
$$

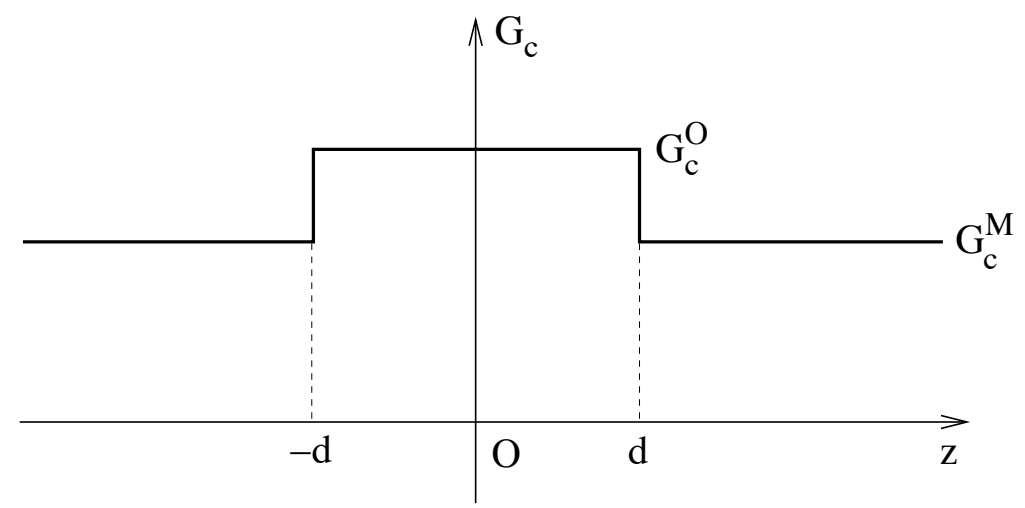

Fig. 3. Distribution of fracture toughness on a plane containing a single infinitely elongated obstacle.

\subsection{Expressions of the crack front shape up to the second order}

The Fourier transform of the function $g_{c}(x, z)$ is given by

$$
\widehat{g_{c}}(x, k) \equiv \widehat{g}_{c}(k)=\frac{1}{2 \pi} \int_{-d}^{d} e^{-i k z} d z=\frac{\sin (k d)}{\pi k} .
$$

At order 1, one gets from equations (35) and (39):

$$
\widehat{\phi^{1}}(a ; k)=-\frac{\sin (k d)}{\pi k\left[2|k| X(k h)-\frac{d G^{0}}{G^{0} d a}(a)\right]}
$$

so that 


$$
\begin{aligned}
\phi^{1}(a ; z) & =-\frac{1}{\pi} \int_{-\infty}^{+\infty} \frac{\sin (k d)}{k\left[2|k| X(k h)-\frac{d G^{0}}{G^{0} d a}(a)\right]} e^{i k z} d k \\
& =-\frac{2}{\pi} \int_{0}^{+\infty} \frac{\sin (k d)}{k\left[2 k X(k h)-\frac{d G^{0}}{G^{0} d a}(a)\right]} \cos (k z) d k
\end{aligned}
$$

In the limit $\frac{d G^{0}}{d a}(a) \rightarrow 0^{-}$, the integral defining $\phi^{1}(a ; z)$ here diverges. However we are interested only in the deviation of the crack front from straightness. This deviation can be characterized at order 1 by the quantity

$$
\widetilde{\phi^{1}}(a ; z) \equiv \phi^{1}(a ; z)-\phi^{1}(a ; 0)=\frac{2}{\pi} \int_{0}^{+\infty} \frac{\sin (k d)}{k\left[2 k X(k h)-\frac{d G^{0}}{G^{0} d a}(a)\right]}[1-\cos (k z)] d k .
$$

This quantity has a well-defined limit $\widetilde{\phi^{1}}(z)$ for $\frac{d G^{0}}{d a}(a) \rightarrow 0^{-}$given by

$$
\widetilde{\phi^{1}}(z) \equiv \frac{1}{\pi} \int_{0}^{+\infty} \frac{\sin (k d)}{k^{2} X(k h)}[1-\cos (k z)] d k
$$

At order 2, equations (36) and (39) yield, since the function $\widehat{g}_{c}(x, k)$ is independent of $x$ :

$$
\begin{array}{rl}
\phi^{2}(a ; z)=\frac{1}{\pi^{2}} \int_{-\infty}^{+\infty} \int_{-\infty}^{+\infty} & S\left(a ; h ; k, k^{\prime}\right) \frac{\sin (k d)}{k\left[2|k| X(k h)-\frac{d G^{0}}{G^{0} d a}(a)\right]} \frac{\sin \left(k^{\prime} d\right)}{k^{\prime}\left[2\left|k^{\prime}\right| X\left(k^{\prime} h\right)-\frac{d G^{0}}{G^{0} d a}(a)\right]} \\
& \times \frac{2}{2\left|k+k^{\prime}\right| X\left(\left(k+k^{\prime}\right) h\right)-\frac{d G^{0}}{G^{0} d a}(a)} d k d k^{\prime} \\
=\frac{\sin (k d)}{\pi^{2}} \iint_{k+k^{\prime} \geq 0} & S\left(a ; h ; k, k^{\prime}\right) \frac{\sin \left(k^{\prime} d\right)}{k\left[2|k| X(k h)-\frac{d G^{0}}{G^{0} d a}(a)\right]} \frac{\cos \left[\left(k+k^{\prime}\right) z\right]}{k^{\prime}\left[2\left|k^{\prime}\right| X\left(k^{\prime} h\right)-\frac{d G^{0}}{G^{0} d a}(a)\right]} \\
\times & \frac{\left.d k^{\prime}\right)}{2\left(k+k^{\prime}\right) X\left(\left(k+k^{\prime}\right) h\right)-\frac{d G^{0}}{G^{0} d a}(a)} d k d k^{\prime}
\end{array}
$$

where we have grouped the terms $\left(k, k^{\prime}\right)$ and $\left(-k,-k^{\prime}\right)$ in the double integral and accounted for the fact that $S\left(a ; h ;-k,-k^{\prime}\right)=S\left(a ; h ; k, k^{\prime}\right)$, see equation (30).

Again, we are interested only in the deviation of the crack front from straightness, characterized at order 2 by the quantity

$$
\begin{aligned}
\widetilde{\phi^{2}}(a ; z) \equiv & \phi^{2}(a ; z)-\phi^{2}(a ; 0)=\frac{2}{\pi^{2}} \iint_{k+k^{\prime} \geq 0} S\left(a ; h ; k, k^{\prime}\right) \frac{\sin (k d)}{k\left[2|k| X(k h)-\frac{d G^{0}}{G^{0} d a}(a)\right]} \\
& \times \frac{\sin \left(k^{\prime} d\right)}{k^{\prime}\left[2\left|k^{\prime}\right| X\left(k^{\prime} h\right)-\frac{d G^{0}}{G^{0} d a}(a)\right]} \frac{\cos \left[\left(k+k^{\prime}\right) z\right]-1}{2\left(k+k^{\prime}\right) X\left(\left(k+k^{\prime}\right) h\right)-\frac{d G^{0}}{G^{0} d a}(a)} d k d k^{\prime} .
\end{aligned}
$$

This quantity has a well-defined limit $\widetilde{\phi^{2}}(z)$ for $\frac{d G^{0}}{d a}(a) \rightarrow 0^{-}$given by

$$
\widetilde{\phi^{2}}(z) \equiv \frac{1}{4 \pi^{2}} \iint_{k+k^{\prime} \geq 0} S^{0}\left(h ; k, k^{\prime}\right) \frac{\sin (k d)}{k|k| X(k h)} \frac{\sin \left(k^{\prime} d\right)}{k^{\prime}\left|k^{\prime}\right| X\left(k^{\prime} h\right)} \frac{\cos \left[\left(k+k^{\prime}\right) z\right]-1}{\left(k+k^{\prime}\right) X\left(\left(k+k^{\prime}\right) h\right)} d k d k^{\prime}
$$


where

$$
\begin{aligned}
S^{0}\left(h ; k, k^{\prime}\right) & \equiv \lim _{d G^{0} / d a \rightarrow 0^{-}} S\left(a ; h ; k, k^{\prime}\right) \\
& =-\frac{1}{2}\left[\left|k+k^{\prime}\right| X\left(\left(k+k^{\prime}\right) h\right)-|k| X(k h)-\left|k^{\prime}\right| X\left(k^{\prime} h\right)\right]^{2}+2|k|\left|k^{\prime}\right| X(k h) X\left(k^{\prime} h\right) .
\end{aligned}
$$

The integral in equation (43) is convergent because the function $S^{0}\left(h ; k, k^{\prime}\right)$ verifies the properties $S^{0}(h ; k, 0)=S^{0}\left(h ; 0, k^{\prime}\right)=0$.

Since the function $S^{0}$ is obviously invariant upon interchange of $k$ and $k^{\prime}$, the integral in Eq. (43) can be simplified by reducing the integration domain $\left\{\left(k, k^{\prime}\right), k+k^{\prime} \geq 0\right\}$ : this domain consists of two sub-domains, where $k \geq k^{\prime}$ and $k^{\prime} \geq k$ respectively, which yield equal contributions; hence the integral is equal to twice the integral over the first subdomain. This sub-domain consists of two sub-sub-domains, $\left\{\left(k, k^{\prime}\right), k \geq 0,0 \leq k^{\prime} \leq k\right\}$ and $\left\{\left(k, k^{\prime}\right), k \geq 0,-k \leq k^{\prime} \leq 0\right\}$; re-noting $k^{\prime}$ as $-k^{\prime}$ in the integral over the second subsub-domain, one finally gets

$$
\begin{aligned}
\widetilde{\phi^{2}}(z)=\frac{1}{2 \pi^{2}} \int_{0}^{+\infty}\left\{\int_{0}^{k}\right. & {\left[S^{0}\left(h ; k, k^{\prime}\right) \frac{\cos \left(\left(k+k^{\prime}\right) z\right)-1}{\left(k+k^{\prime}\right) X\left(\left(k+k^{\prime}\right) h\right)}\right.} \\
& \left.\left.+S^{0}\left(h ; k,-k^{\prime}\right) \frac{\cos \left(\left(k-k^{\prime}\right) z\right)-1}{\left(k-k^{\prime}\right) X\left(\left(k-k^{\prime}\right) h\right)}\right] \frac{\sin (k d)}{k^{2} X(k h)} \frac{\sin \left(k^{\prime} d\right)}{k^{\prime 2} X\left(k^{\prime} h\right)} d k^{\prime}\right\} d k .
\end{aligned}
$$

One may now write $k^{\prime} \equiv \lambda k, 0 \leq \lambda \leq 1$ and use the variables of integration $(k, \lambda)$ instead of $\left(k, k^{\prime}\right)$; the preceding equation then becomes

$$
\begin{aligned}
\widetilde{\phi^{2}}(z)=\frac{1}{2 \pi^{2}} \int_{0}^{+\infty}\left\{\int_{0}^{1}[\right. & S^{0}(h ; k, \lambda k) \frac{\cos ((1+\lambda) k z)-1}{(1+\lambda) X((1+\lambda) k h)} \\
& \left.\left.+S^{0}(h ; k,-\lambda k) \frac{\cos ((1-\lambda) k z)-1}{(1-\lambda) X((1-\lambda) k h)}\right] \frac{\sin (k d)}{X(k h)} \frac{\sin (\lambda k d)}{\lambda^{2} X(\lambda k h)} d \lambda\right\} \frac{d k}{k^{4}} .
\end{aligned}
$$

Introducing the notation

$$
\frac{S^{0}(h ; k, \lambda k)}{k^{2} X(k h) X(\lambda k h) X((1+\lambda) k h)} \equiv T^{0}(k h, \lambda)
$$

in the preceding integral and changing the order of integration, one gets the final expression of $\widetilde{\phi^{2}}(z)$ :

$$
\begin{aligned}
\widetilde{\phi^{2}}(z)=\frac{1}{2 \pi^{2}} \int_{0}^{1} & \left\{\frac{J[h ; \lambda ; d, \lambda d,(1+\lambda) z]-J(h ; \lambda ; d, \lambda d, 0)}{1+\lambda}\right. \\
& \left.+\frac{J[h ;-\lambda ; d, \lambda d,(1-\lambda) z]-J(h ;-\lambda ; d, \lambda d, 0)}{1-\lambda}\right\} \frac{d \lambda}{\lambda^{2}}
\end{aligned}
$$

where

$$
J(h ; \lambda ; \alpha, \beta, \gamma) \equiv \int_{0}^{+\infty} T^{0}(k h, \lambda) \sin (\alpha k) \sin (\beta k) \cos (\gamma k) \frac{d k}{k^{2}}
$$




\subsection{Limiting cases of infinite and infinitesimal thickness}

In the limit of an infinite body $(h \rightarrow+\infty)$, for which $X(k h) \rightarrow \frac{1}{2}$, the integrals in Eqs. (41), (46) and (47) may be calculated analytically, and the final results for $\widetilde{\phi^{1}}$ and $\widetilde{\phi^{2}}$ read (Chopin et al., 2011; Vasoya et al., 2013):

$$
\begin{cases}\lim _{h \rightarrow+\infty} \widetilde{\phi^{1}}(z)=\frac{d}{\pi}[(1+u) \ln (|1+u|)+(1-u) \ln (|1-u|)] & \\ \lim _{h \rightarrow+\infty} \widetilde{\phi^{2}}(z)= \begin{cases}-\frac{d}{2 \pi}[(1+u) \ln (1+u)+(1-u) \ln (1-u)] & \text { if }|u| \leq 1 \quad, \quad u \equiv \frac{z}{d} \\ -\frac{d}{2 \pi}\left[(|u|-1) \ln \left(\frac{|u|+1}{|u|-1}\right)+2 \ln 2\right] & \text { if }|u| \geq 1\end{cases} \end{cases}
$$

Note that for $u= \pm 1$, the values of the functions here are defined only as limits using the property $\lim _{x \rightarrow 0^{+}} x \ln x=0$.

On the other hand, for an infinitely thin plate $(h \rightarrow 0), X$ is multiplied by a factor of 4 with respect to the case $h \rightarrow+\infty$, so that by Eqs. (41), (44), (45), (46) and (47), $\widetilde{\phi^{1}}$ and $\widetilde{\phi^{2}}$ are simply divided by a factor of 4 ; that is,

$$
\begin{cases}\lim _{h \rightarrow 0} \widetilde{\phi^{1}}(z)=\frac{d}{4 \pi}[(1+u) \ln (|1+u|)+(1-u) \ln (|1-u|)] & \\ \lim _{h \rightarrow 0} \widetilde{\phi^{2}}(z)= \begin{cases}-\frac{d}{8 \pi}[(1+u) \ln (1+u)+(1-u) \ln (1-u)] & \text { if }|u| \leq 1 \\ -\frac{d}{8 \pi}\left[(|u|-1) \ln \left(\frac{|u|+1}{|u|-1}\right)+2 \ln 2\right] & \text { if }|u| \geq 1\end{cases} \end{cases}
$$

\subsection{Numerical solutions for arbitrary values of the thickness}

For arbitrary values of $h$, the integrals appearing in Eqs. (41), (46) and (47) may be calculated numerically. Instead of plotting both $\widetilde{\phi^{1}}(z)$ and $\widetilde{\phi^{2}}(z)$, we choose to plot the sole perturbation of the front defined by: ${ }^{2}$

$$
\delta a(z)=\epsilon \widetilde{\phi^{1}}(z)+\epsilon^{2} \widetilde{\phi^{2}}(z)
$$

In the following figures, aiming at validating these numerical calculations, its normalized value is displayed for $\varepsilon=1$.

Figure 4 first compares, in the two limiting cases $h \rightarrow+\infty$ and $h \rightarrow 0$, the values of $\frac{\delta a(z)}{d}$ obtained numerically (full lines) and analytically (dotted lines). The agreement is excellent in both cases, which shows that the numerical calculation of the integrals is correct and accurate.

$\overline{2}$ This definition differs from that used in Section $4, \epsilon \phi^{1}(z)+\epsilon^{2} \phi^{2}(z)$, through the quantity $\epsilon \phi^{1}(0)+\epsilon^{2} \phi^{2}(0)$. The new definition means that the perturbation of the crack front is observed using a new reference straight line passing through the point $z=0$ of the perturbed front. It will also be used in Section 6.4 below. 


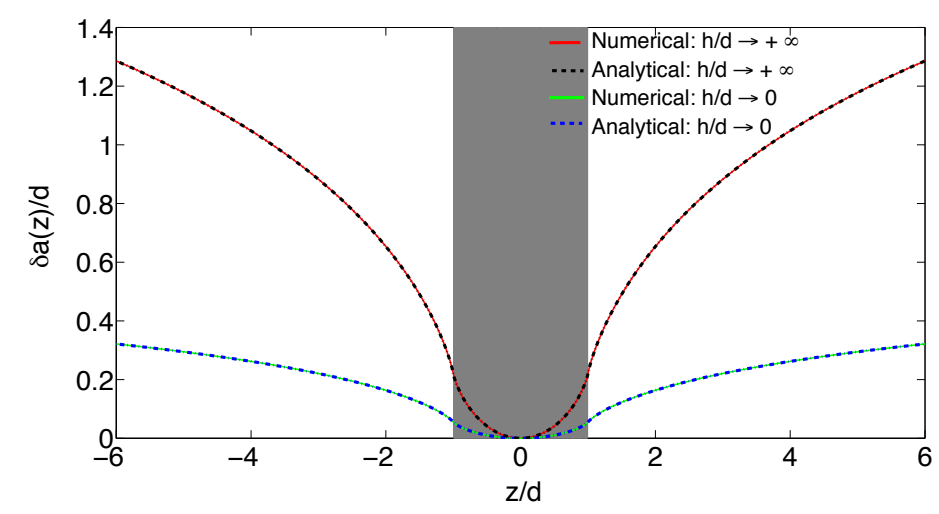

Fig. 4. Equilibrium shape of a crack front penetrating into a single obstacle of normalized toughness contrast $\epsilon=1$, in plates of infinite and infinitesimal thicknesses.

Figure 5 now shows the same quantity as a function of the normalized plate thickness $h / d$, for the same normalized toughness contrast $\epsilon$ of unity. The dependence of $\delta a(z) / d$ upon $h / d$ is quite clear here: when the plate becomes thinner, the deformation of the crack front decreases, that is, this front becomes "stiffer". Note that although the value $\epsilon=1$ here is admittedly high, the perturbation of the front remains reasonably small.

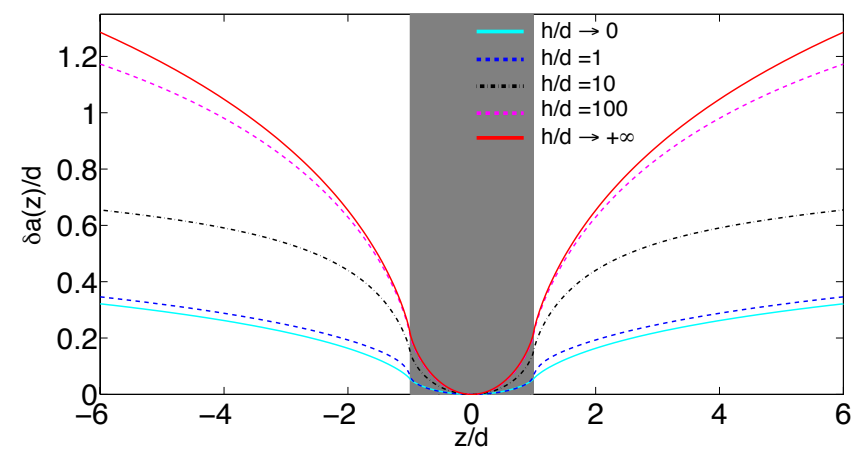

Fig. 5. Equilibrium shape of a crack front penetrating into a single obstacle of normalized toughness contrast $\epsilon=1$, in plates of various thicknesses.

It is worth noting that the effect of the finite thickness of the plate on the crack front geometry is more pronounced in the thick plate regime, $h \gg d$, than in the thin plate regime, $h \ll d$. Indeed the solution for an infinitely thick plate provides an acceptable approximation (within $10 \%$ in the domain $|z / d| \leq 6$ ) only for values of $h / d$ exceeding 100, whereas that for an infinitely thin plate remains acceptable (with the same error on the same domain) up to values of $h / d$ of the order of 0.4 . This observation suggests that the finite thickness of the specimen may have had a significant impact on the experimental front geometries reported in the works of Delaplace et al. (1999) and Dalmas et al. (2008).

Another interesting feature is that the range of validity of the first order approximation is independent of the specimen thickness, and extends (within $10 \%$ for all values of $z$ ) up to contrasts $\epsilon \simeq 0.2$. Indeed, this range of validity may be estimated from the ratio of the first-order contribution to the second-order one, and this ratio is the same for infinitely thin and thick plates, see Eqs. (48) and (49). One of the aims of the following section is to study experimentally this range of validity. 


\section{Experimental investigation of crack pinning by strong designed obstacles}

We shall now explore experimentally the process of crack pinning by strong heterogeneities. We shall focus on the infinitely thin plate limit $h / d \rightarrow 0$, and investigate the relevance of the second-order formula derived in the previous section to describe the deformation of cracks due to a single obstacle. For this, we investigate experimentally the peeling of a thin film (F) from a patterned substrate (S) (see fig. 6).

\subsection{Experimental setup}
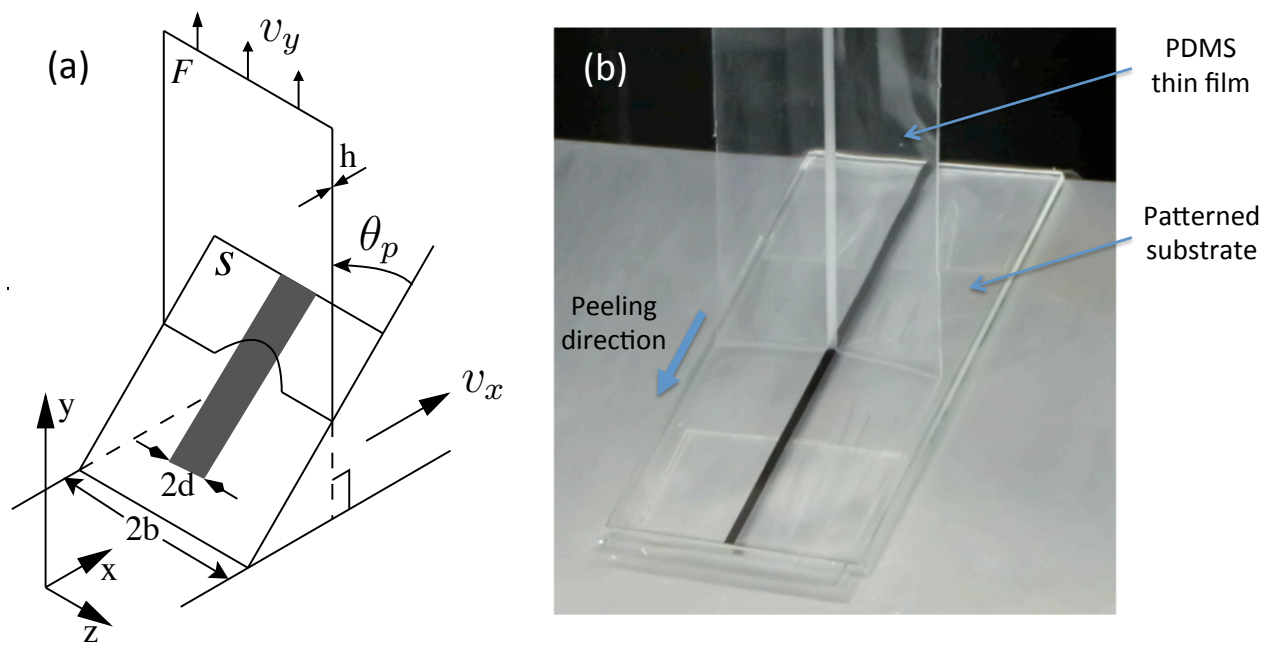

Fig. 6. Experimental setup: (a) Schematic representation of the peeling test. The substrate is moved horizontally at the velocity $v_{x}$ in order to maintain a constant peeling angle $\theta_{p}$. (b) Snapshot of the experiment.

The specimen preparation follows the procedure detailed in Xia et al. (2012). To introduce an obstacle of controlled geometry and toughness and permit a quantitative comparison with the predictions of Section 5 , an obstacle of width $2 d$ aligned with the peeling direction is printed on a transparency using a standard commercial printer. The neat side of the transparency is subsequently glued onto a glass plate to form the substrate. The thin film is then spin-coated directly over the printed face from a liquid solution of PDMS obtained by mixing an elastomer and a curing agent of PDMS (Sylgard 184, Dow Corning Co.) at a weight ratio of 10:1 by spinning the machine at a rate of $300 \mathrm{rpm}$ during $20 \mathrm{~s}$. This procedure produces homogeneous thin films with thickness $h=400 \mu \mathrm{m}$. This phase is followed by curing of the thin film and its substrate at a temperature of $60^{\circ} \mathrm{C}$ for two hours. Both curing temperature and time control the cross linking of PDMS and lead to a Young's modulus $E$ and a Poisson's ratio $\nu$ of $1.2 \mathrm{MPa}$ and 0.5 (a value characteristic of incompressible elastomers), respectively. The heat treated samples are then kept at room temperature for 48 hours prior to the test. This two-stage curing treatment is used to minimize the residual stress in cured PDMS.

A standard peel test configuration is employed to measure the peel force required to peel off the PDMS thin film. As shown in Fig. 6, the free end of the PDMS thin film is peeled 
off at a constant speed, $v_{p}=1.65 \mathrm{~mm} . \mathrm{s}^{-1}$, and at a peel angle $\theta_{p}=45^{\circ}$, from the inkpatterned rigid substrate. For ease of experimental implementation, the film is always peeled vertically, and the rigid substrate is rotated about its hinge to obtain any desired peel angle. In order to maintain a constant peel angle $\theta_{p}$ during the test, the substrate is moved in the horizontal direction at a speed $v_{x}=v_{y} \sin \theta_{p} /\left(1-\cos \theta_{p}\right)$. In this way, a stationary regime of propagation is reached.

\subsection{Compatibility with the theoretical framework}

The defect width $2 d$ is chosen to be small enough so that $d \ll 2 \pi r_{b}$ where the bending length $r_{b}=\sqrt{E h^{3} /\left[24\left(1-\nu^{2}\right) \overline{G_{c}}\right]}$ represents the thin film's radius of curvature near the peeling front, but large enough so that $d \gtrsim h$. The first condition ensures that the nonlinear effects arising from the large displacements of the film can be neglected and the LEFM approach developed in the previous sections applies (Xia et al., 2015). The second condition ensures that the geometry of the detachment front can be described by the previous calculations in the limit $h / d \rightarrow 0$. In practice, $d=1 \mathrm{~mm}$ while $r_{b}=1.4 \mathrm{~mm}$, so that $h / d=0.4$ and $d /\left(2 \pi r_{b}\right) \simeq 0.1$. Finally, the choice of a relatively large peeling angle, $\theta_{p}=45^{\circ}$, is motivated by the occurrence of large stretching deformations for small peeling angles that dominate over the bending mode of deformation of the film considered in our calculation. This transition from a peeling mode governed by stretching to a mode governed by bending occurs for a peeling angle $\theta_{p}^{c} \simeq \sqrt[4]{\overline{G_{c}} / E h} \simeq 15^{\circ}$ (Ponson et al., in preparation).

It is now necessary to explain why, in the limit of very thin plates $h / d \rightarrow 0$, the LEFM analysis of a crack lying at the interface between two elastic plates (see Fig. 2(b)) applies to the peeling of an elastic plate from a rigid substrate. It so occurs that for the problem considered in Fig. 2(b), the replacement of the bottom elastic plate by a rigid one does not affect the calculation, as long as the limit $h / d \rightarrow 0$ is considered. Indeed in this limit, as a result of the Love-Kirchhoff theory of thin plates, all components of the displacement are identically zero on the unbroken part of the interface between the two plates, irrespectively of the elasticity of the bottom plate; hence the boundary conditions imposed onto the top plate by the bottom one are exactly the same as if the latter were rigid ${ }^{3}$. Of course, for a given front geometry, the perturbation $\delta G$ of the elastic energy-release-rate is twice larger when the upper plate is bonded onto another elastic plate rather than on some rigid substrate, since the same amount of elastic energy is released from both plates; but since the same argument also holds for the elastic energy-release-rate $G^{0}$ for a straight crack front, Eqs. (49) derived above using only the value of the ratio $\delta G / G^{0}$ apply indifferently to both cases.

$\overline{3}$ This does not remain true for elastic plates of finite thickness, because as a result of the theory of $3 \mathrm{D}$ elasticity, the horizontal components of the displacement are no longer zero on the unbroken part of the interface. 


\subsection{Characterization of the adhesion energies}

To introduce defects pinning the detachment front, we take advantage of the strong adhesion of PDMS on ink, characterized by the high interfacial fracture energy $G_{c}^{\mathrm{O}}$, as compared to that on neat transparency, characterized by the lower energy $G_{c}^{\mathrm{M}}$. To vary the strength of these defects, we tune their grayscale $c_{\text {gray }}\left(c_{\text {gray }}=0\right.$ without any obstacle and $c_{\text {gray }}=1$ for a black obstacle).

The first task is to measure the interfacial fracture energies of PDMS with the transparency $\left(G_{c}^{\mathrm{M}}\right)$, and with the printed obstacle $\left(G_{c}^{\mathrm{O}}\right)$ as a function of $c_{\text {gray }}$. To do so, we first deduce the mean interfacial fracture energy $\overline{G_{\mathrm{c}}}$ from Rivlin (1944)'s equation

$$
\overline{G_{c}}=\frac{F_{p}}{2 b}\left(1-\cos \theta_{p}\right)
$$

where $F_{p}$ is the steady-state peel force measured and $b$ (=24 $\mathrm{mm}$ in the tests) the halfwidth of the adhesive ${ }^{4}$ (see Fig. 6). An alternative, more refined equation due to Kendall (1973) incorporating the membrane elasticity of the film is actually used in the following, without significantly changing the results obtained (see Fig. 7). The inset (a) of Fig. 7 shows the peel force $F_{p}$ as a function of the peel displacement, for various values of the width $2 d$ of the obstacle. One sees that the force quickly increases to reach a stationary value. This reflects the stationary loading conditions imposed to the adhesive that is peeled with an angle maintained constant during the experiment.

To now deduce the values of the toughness contrast $\epsilon=\frac{G_{c}^{\mathrm{O}}\left(c_{\text {gray }}\right)-G_{c}^{\mathrm{M}}}{G_{c}^{\mathrm{M}}}$ from that of $\overline{G_{c}}$, we perform peel strength measurements of samples with defects with the same gray level $c_{\text {gray }}$ but various widths $2 d$, and use the equation

$$
\overline{G_{c}}=\left(1-\frac{d}{b}\right) G_{c}^{\mathrm{M}}+\frac{d}{b} G_{c}^{\mathrm{O}} \Rightarrow \frac{\overline{G_{c}}}{G_{c}^{\mathrm{M}}}=1+\frac{d}{b} \epsilon
$$

expressing the "mixture rule" for the effective fracture energy of heterogeneous interfaces invariant along the propagation direction. This procedure is illustrated in Fig. 7 where the normalized effective fracture energy $\overline{G_{c}} / G_{c}^{\mathrm{M}}$ is plotted as a function of the normalized defect width $d / b$. The slope of the linear fit provides the fracture energy contrast $\epsilon=0.66 \pm$ 0.06 where the uncertainty is determined from the quality of the fit and the uncertainty on the fracture energies (see Appendix B). The adhesion energy $G_{c}^{\mathrm{M}}=2.21 \pm 0.02 \mathrm{~J} . \mathrm{m}^{-2}$ of the PDMS-transparency is measured independently through additional peel tests performed on homogeneous specimens. Note that the values of fracture energy are found to slightly vary with the velocity of the peeling front. In the experiments reported in this paper, the velocity is therefore fixed to a value of $v_{\mathrm{p}}=1.65 \mathrm{~mm} \cdot \mathrm{s}^{-1}$.

The very same procedure is applied for various grey levels, so that the contrast $\epsilon$ can be measured as a function of $c_{\text {gray }}$, as shown in the inset (b) of Fig. 7 . One observes that

$\overline{4}$ This width is kept constant during the first set of experiments discussed here, but will be varied in a second set discussed in Section 6.5 below. 


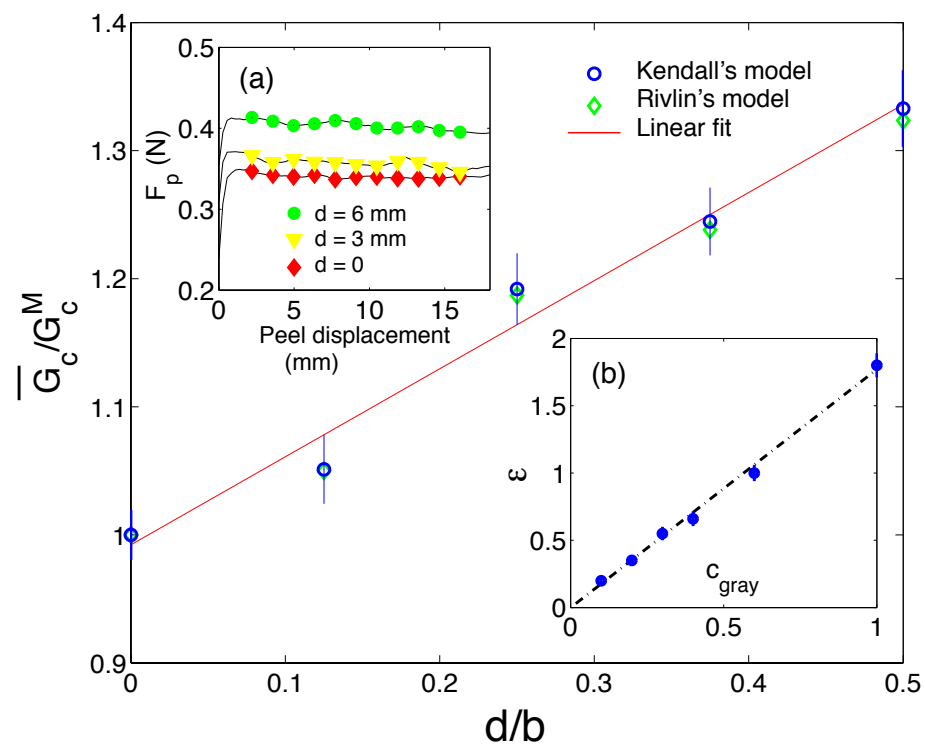

Fig. 7. Normalized effective adhesion energy of the interface as a function of the normalized width $d / b$ of the obstacle for $c_{\text {gray }}=0.4$. The slope of the linear fit gives the toughness contrast $\epsilon=0.66 \pm 0.06$. Inset (a): Peel force versus displacement curves for the same grayscale but different obstacle half-widths. Inset (b): Contrast of interfacial fracture energy versus grayscale of the obstacle.

$\epsilon\left(c_{\text {gray }}\right)$ evolves linearly with $c_{\text {gray }}$, according to the law $\epsilon\left(c_{\text {gray }}\right)=1.77 c_{\text {gray }}$. The range $0 \leq \epsilon \leq 1.77$ of contrasts achieved by our experimental setup allows to study crack front pinning beyond the domain of validity of the first-order theory and to investigate the relevance of the second-order correction.

\subsection{Deformation of the front pinned by obstacles of varying strength}

A digital camera equipped with a high-magnification lens is positioned above the substrate surface, perpendicularly to it, and used to record in situ images of the peel-front configuration. An example of the picture obtained is shown in Fig. 8(c). Using an edequate lighting device, the front shape appears as a thin bright line. The other, thicker bright line that appears on the raw image results from the reflection of the light on the curvy part of the debonded thin film and is discarded in the analysis. Image processing is then used to enhance the contrast and discrete positions of the front are obtained by manual selection on a zoomed image. In the stationary experimental regime, $G_{0}$ does not depend on the crack size $a$, hence it is not possible to define a mean position of the front using Eq. (34). Instead, from now on, we take as reference the straight line which intersects the front at $z=0$ to define the crack front perturbation $\delta a$, in agreement with Eq. (50). The result is plotted on Fig. 8(a) with an amplification in the $y$-direction. One immediately recognizes the characteristic shape of a crack front pinned by an isolated obstacle of larger toughness, as has been observed by Dalmas et al. (2008); Patinet et al. (2013a); Budzik et al. (2013); Chopin et al. (2011). 

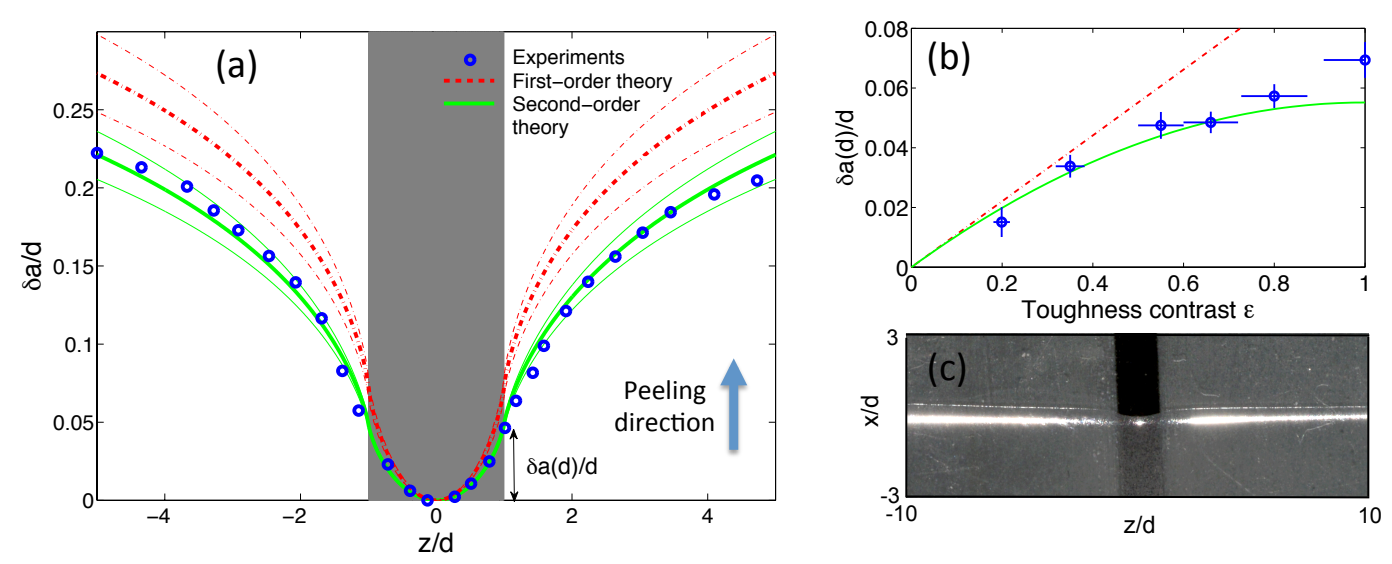

Fig. 8. (a) Equilibrium shape of a crack front pinned by a single obstacle of contrast $\epsilon=0.66 \pm 0.06$, and comparison with the first- and second-order theoretical predictions (Eqs. (49)). (b) Variations of the amplitude $\delta a(d) / d$ of the crack front deformation as a function of the contrast, and comparison with the predictions of the first- and second-order theories. (c) Typical snapshot of the peeling front as it crosses an obstacle.

The first- and second-order approximations (Eqs. (49)), corresponding to the limit $h / d \rightarrow$ 0 , are also plotted in Fig. 8(a) using the contrast value $\epsilon=0.66$ measured independently from peel force measurements. To take into account the experimental uncertainties on the contrast value, we also plot in thinner lines the lower and upper bounds of the theory that correspond to $\epsilon^{\min }=0.60$ and $\epsilon^{\max }=0.72$, respectively. It is observed that the second-order theory brings a significant correction to the first-order one, by predicting a less deformed ("stiffer") crack front, and considerably improves the agreement with the experiment.

The "tunability" of the printed obstacles may be exploited to explore other contrasts. Fig. 8(b) shows the normalized amplitude of the crack front perturbation, defined as the amplitude of this deformation within the obstacle, that is the difference of position $\delta a(d)$ of the front at the edge and center of this obstacle, normalized by the defect half-width $d$. This quantity depends only on the contrast, and varies linearly with it according to the first-order theory. In contrast, the experiments exhibit some "saturation" of the amplitude as the contrast increases. This saturation is correctly predicted by the second-order theory.

This quantitative comparison with experiments permits to define the practical limits of the first- and second-order theories. We retrieve experimentally that the first-order calculation provides an accurate prediction of the deformation of the front of a pinned crack for low contrasts, $\epsilon \lesssim 0.2-0.3$. It also provides the domain of validity of the secondorder calculation that describes accurately the experimental results for $\epsilon \lesssim 0.7$. For larger contrasts, the second-order theory underestimates the deformation of the front; either a higher-order development or a numerical approach (see Bower and Ortiz (1991); Lazarus (2003)) would be needed to accurately describe the geometry of the crack front in such situations. 


\subsection{Effect of the sample width on the deformation of the front}

In the previous set of experiments, we limited our analysis to the region $|z / d| \leq 5$. Beyond this domain, we observe deviations from the theoretical predictions that suggest that the effect of the finite width neglected in the calculations may begin to play a significant role.

To investigate this question, we measure the crack front deformation for a fixed contrast $\epsilon=0.66$ and a fixed defect half-width $d=1 \mathrm{~mm}$, but various specimen widths $2 b$. Figure 9 (a) shows the results obtained. ${ }^{5}$ The comparison with the first- and second-order theories, strictly valid in the limit $b / d \rightarrow+\infty$, is also displayed in Fig. 9(a). It shows that the specimen width has a negligible effect on the crack front deformation in the region of interest $|z|<5 d$ as long as $b \gtrsim 12 d$. Provided this condition is met, the second-order expansion captures the front geometry fairly well, confirming the results of the previous section and the relevance of the second-order correction for stronger obstacles.
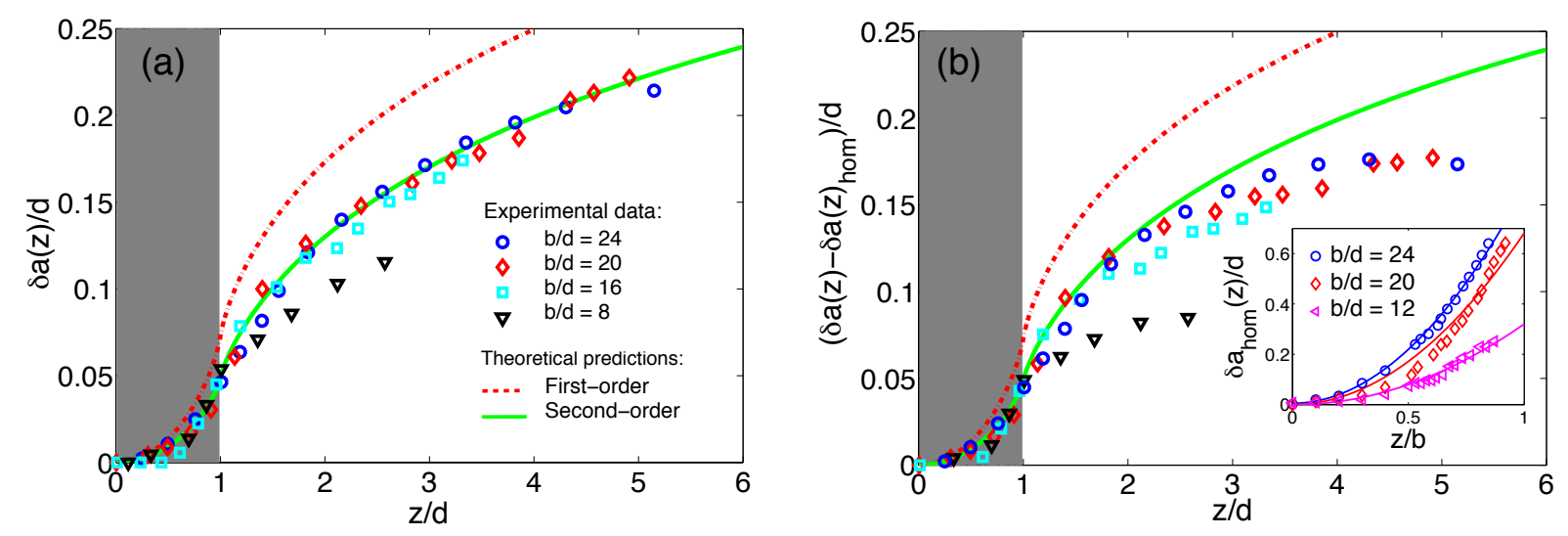

Fig. 9. Effect of the specimen width on the front perturbation: (a) Geometry of the pinned front for $\epsilon=0.66$ for various specimen widths 2b. (b) Geometry of the pinned front after correction by the unpinned front geometry.

For the smallest sample having $b / c=8$, the crack front geometry is clearly influenced by the finite width of the specimen. To account for this effect, Patinet et al. (2013a) proposed to correct the crack front deformation $\delta a(z)$ observed by subtracting the deformation $\delta a_{\text {hom }}(z)$ measured for a homogeneous interface, before comparing it to that predicted theoretically for an infinitely wide specimen. This procedure is tentatively applied to our experiments: First, the crack front geometry is measured in homogeneous specimens of various widths $2 b$, as shown by the open symbols in the inset of Fig. 9(b). A parabolic fit $\delta a_{\text {hom }}(z)=0.037 z^{2} / b$ shown by continuous lines provides an accurate description of the experimental points. This deformation is then subtracted from the original deformation and the corrected crack front geometry is compared to the theoretical predictions in Fig. 9(b). One observes that this procedure actually degrades the agreement between experimental and theoretical curves.

5 Because of the symmetry $(z \rightarrow-z)$ of the peeling test geometry, we only show one half of the front and represent the mean value of the deformations $\delta a(z)$ measured in the regions $z<0$ and $z>0$. 
The apparent inappropriateness of this procedure might relate to the implicit assumption that the zeroth-order crack front shape corresponding to a homogeneous interface is influenced by the finite width of the specimen, but that the first- and second-order corrections due to the presence of the obstacle are not. There is no clear reason why this assumption should be correct, especially considering the large influence of the specimen thickness $h$ upon the first- and second-order corrections.

A discussion of the results of Patinet et al. (2013a) is finally possible. These authors performed similar measurements of the crack front deformation when pinned by a single obstacle, and concluded that the predictions of the first-order theory were quantitatively correct over a much larger range of values of the contrast than found here. Since this range of validity was shown to be independent of the specimen thickness, we might attribute this discrepancy to the finite width of the specimen: Patinet et al. (2013a) used specimen widths in the range $9 \lesssim b / d \lesssim 13$, for which the effect of the finite width of the specimen was significant in our setup, and used the procedure of correction depicted above. Now in the double cantilever beam they used, the sign of the curvature of the crack front for a homogeneous interface is opposite to that in our peeling test. As a result, the subtraction of $\delta a_{\text {hom }}(z)$ from $\delta a(z)$ resulted in an increase of the crack front deformation instead of a decrease like in our case (see Figs. 9(a) and (b)). If, as suggested by the above results, the procedure of correction was in reality inappropriate, it might have led to an overestimation of the crack front deformation bringing it closer to the predictions of the first-order theory, and thus giving the impression of correctness of this theory beyond its real range of applicability.

While the effect of the specimen thickness on the pinning of crack fronts can now be quantitatively taken into account, the role played by the specimen width is less obvious and deserves further investigations. To circumvent this difficulty in our experiments, we used thin films of sufficiently large width $b \gtrsim 12 d$ with respect to the obstacle width. Note however that this range of validity might vary with the test geometry employed.

\section{Conclusion}

The primary objective of this work was to provide a quantitative description of the process of crack pinning by heterogeneities (i) taking into account the finite size of the specimens and (ii) going beyond the linear first order approximation.

The problem was addressed theoretically by calculating the second-order expansion of the stress intensity factor resulting from some small, but otherwise arbitrary coplanar perturbation of the front of a semi-infinite crack lying on the mid-plane of a plate of finite thickness and subjected to general mode I loading conditions. The results obtained were used to predict the equilibrium geometry of the crack for a given slightly heterogeneous distribution of fracture energy within its plane. Specializing to the pinning by a single infinitely elongated obstacle, we found a formula for the shape of the crack front accurate to second order in the toughness contrast. This formula was fully explicit in all cases, and entirely analytic (i.e. did not require the numerical evaluation of any integrals) in the two 
extreme limits of infinitely thick and infinitely thin plates.

The remainder of the paper focused on experiments performed in the latter limit, using peeling tests with fully controlled fields of local fracture energy. The experimental crack front deformations were observed to be in good agreement with the theoretical formulas and the second order term to (i) greatly improve the agreement and (ii) broaden their range of applicability in terms of admissible values of the toughness contrast.

This work highlights the following generic features of crack pinning in samples of finite size:

- For a given distribution of obstacles, the thinner the sample, the smaller the deformation of the front. In the limit of a very thin specimen, this deformation is exactly four times smaller than in the limit of an infinitely thick one.

- The second-order correction relevant for large toughness contrasts shows that the crack front stiffens under the action of strong obstacles; in other words, the first-order approximation overestimates the deformation of the crack pinned by such obstacles. This approximation is sufficient for normalized contrasts up to $\epsilon \simeq 0.2$, whereas the secondorder one correctly predicts the crack front deformation up to $\epsilon \simeq 0.7$, irrespective of the specimen thickness.

- Although the effect of specimen thickness can now be quantitatively taken into account, the finite width of the specimen might play a significant role and deserve further investigations. In practice, we found that in peeling tests, films at least 12 times wider than the defect size are necessary in order to apply the theory developed in this paper under the hypothesis of infinite width.

- Generally speaking, the non-linear correction arising from large contrasts of toughness is expected (Delaplace et al., 1999; Xia et al., 2012) to play a significant role in various experimental situations and should be taken into account to predict the failure behavior of engineered heterogeneous materials with new and improved properties. We have shown that the second order approximation is useful to increase the accuracy. However numerical methods become unavoidable to consider strong heterogeneities. An iterated perturbation approach is then a promising method (Vasoya et al., 2014).

\section{Acknowledgements}

The support of the European Union throughout a Marie Curie integration grant "ToughBridge" (LP), of the city of Paris throughout the Emergence program (LP) and of the ANR Program SYSCOMM (ANR-09-SYSC-006) (VL), is gratefully acknowledged. We also wish to thank Damien Vandembroucq and Sylvain Patinet for stimulating discussions. 


\section{References}

Bonamy, D., Bouchaud, E., 2011. Failure of heterogeneous materials: a dynamic phase transition? Phys. Rep. 498, 1-44.

Bonamy, D., Santucci, S., Ponson, L., 2008. Crackling dynamics in material failure as the signature of a self-organized dynamic phase transition. Phys. Rev. Lett. 101, 045501.

Bower, A. F., Ortiz, M., 1991. A 3-dimensional analysis of crack trapping and bridging by tough particles. J. Mech. Phys. Solids 39, 815-858.

Budzik, M. K., Jumel, J., Shanahan, M. E. R., 2013. Adhesive fracture of heterogeneous interfaces. Phil. Mag. 93, 2413-2427.

Chopin, J., Prevost, A., Boudaoud, A., Adda-Bedia, M., 2011. Crack front dynamics across a single heterogeneity. Phys. Rev. Lett. 107, 144301.

Dalmas, D., Barthel, E., Vandembroucq, D., 2010. Crack front pinning by design in planar heterogeneous interfaces. J. Mech. Phys. Solids 57, 446-457.

Dalmas, D., Lelarge, A., Vandembroucq, D., 2008. Crack propagation through phaseseparated glasses: effect of the characteristic size of disorder. Phys. Rev. Lett. 101, 255501.

Delaplace, A., Schmittbuhl, J., Måløy, K. J., 1999. High resolution description of a crack front in a heterogeneous plexiglas block. Phys. Rev. E 60, 1337-1343.

Démery, V., Rosso, A., Ponson, L., 2014. From microstructural features to effective toughness in disordered brittle solids. Europhys. Lett. 105, 34003.

Gao, H., Rice, J. R., 1989. A first-order perturbation analysis of crack trapping by arrays of obstacles. ASME J. Appl. Mech. 56, 828-836.

Kendall, K., 1973. Thin film peeling-elastic term. J. Phys. D 8, 105-117.

Lazarus, V., 2003. Brittle fracture and fatigue propagation paths of 3D plane cracks under uniform tensile loading. Int. J. Frac. 122, 33-46.

Lazarus, V., 2011. Perturbation approaches of a planar crack in linear elastic fracture mechanics: a review. J. Mech. Phys. Solids 59, 121-144.

Leblond, J.B., Patinet, S., Frelat, J., Lazarus, V., 2012. Second-order coplanar perturbation of a semi-infinite crack in an infinite body. Engng. Frac. Mech. 90, 129-142.

Legrand, L., Patinet, S., Leblond, J.B., Frelat, J., Lazarus, V., Vandembroucq, D., 2011. Coplanar perturbation of a crack lying on the mid-plane of a plate. Int. J. Frac. 170, $67-82$.

Patinet, S., Alzate, L., Barthel, E., Dalmas, D., Vandembroucq, D., Lazarus, V., 2013a. Finite size effects on crack front pinning at heterogeneous planar interfaces: experimental, finite elements and perturbation approaches. J. Mech. Phys. Solids 61, 311-324.

Patinet, S., Vandembroucq, D., Roux, S., 2013b. Quantitative prediction of effective toughness at random heterogeneous interfaces. Phys. Rev. Lett. 110, 165507.

Ponson, L., Bonamy, D., 2010. Crack propagation in brittle heterogeneous solids: material disorder and crack dynamics. Int. J. Frac. 162, 21-31.

Ponson, L. et al. (in preparation). Effect of peeling angle on the adhesion of heterogeneous thin films.

Ramanathan, S., Ertas, D., Fisher, D. S., 1997. Quasistatic crack propagation in heterogeneous media. Phys. Rev. Lett. 79, 873-876.

Rice, J. R., 1985. First-order variation in elastic fields due to variation in location of a planar crack front. ASME J. Appl. Mech. 52, 571-579.

Rice, J. R., 1989. Weight function theory for three-dimensional elastic crack analysis. 
In: Wei, Gangloff (Eds.), Fracture mechanics: Perspectives and directions, ASTM STP 1020. American Society for Testing and Materials, Philadelphia, pp. 29-57.

Rivlin, R. S., 1944. The effective work of adhesion. Paint Technol. 9, 215-218.

Santucci, S., Grob, M., Toussaint, R., Schmittbuhl, J., Hansen, A., Måløy, K. J., 2010. Fracture roughness scaling: a case study on planar cracks. Europhys. Lett. 92, 44001.

Schmittbuhl, J., Delaplace, A., Måløy, K. J., Perfettini, H., Vilotte, J. P., 2003. Slow crack propagation and slip correlations. Pure Appl. Geophys. 160, 396-976.

Schmittbuhl, J., Roux, S., Vilotte, J. P., Måløy, K. J., 1995. Interfacial crack pinning: Effect of nonlocal interactions. Phys. Rev. Lett. 74, 1787-1790.

Vasoya, M., Leblond, J.B., Ponson, L., 2013. A geometrically nonlinear analysis of coplanar crack propagation in some heterogeneous medium. Int. J. Solids Structures 50, $371-378$.

J. R. Taylor, 1997. An introduction to error analysis: The study of uncertainties in physical measurements. University Science Books, Sausalito, CA, USA.

Vasoya, M., Lazarus, V. , Ponson, L., 2014. Crack Front Fingering During Planar Crack Propagation in Highly Heterogeneous Toughness Field. Procedia Materials Science 3, $2142-2147$.

Willis, J. R. , 2013. Crack front perturbations revisited. Int. J. Frac. 184, 17-24.

Willis, J. R., Movchan, N. V. 2014. Second order in-plane dynamic perturbation of a crack propagating under shear loading. Math. Mech. Solids 19, 82-92.

Xia, S., Ponson, L., Ravichandran, G., Bhattacharya, K., 2012. Toughening and asymmetry in peeling of heterogeneous adhesives. Phys. Rev. Lett. 108, 196101.

Xia, S., Ponson, L., Ravichandran, G., Bhattacharya, K., 2015. Adhesion of heterogeneous thin films: II. Adhesive heterogeneity. J. Mech. Phys. Solids 83, 88-103.

\section{A Appendix : Calculation of integrals $I_{1}$ and $I_{2}$}

\section{A.1 Integral $I_{1}$}

This integral is given by

$$
\begin{aligned}
I_{1}\left(h ; k, k^{\prime} ; z_{1}\right)=P V \int_{-\infty}^{+\infty} \int_{-\infty}^{+\infty} & \frac{f\left(\frac{z-z_{1}}{h}\right) f\left(\frac{z^{\prime}-z}{h}\right)}{\left(z-z_{1}\right)^{2}\left(z^{\prime}-z\right)^{2}} \\
& \times\left(e^{i k\left(z-z_{1}\right)}-1\right)\left(e^{i k^{\prime}\left(z^{\prime}-z\right)}-1\right) e^{i k^{\prime}\left(z-z_{1}\right)} d z d z^{\prime} .
\end{aligned}
$$

Quite remarkably, one may express it in terms of the sole function $X$ defined by Eq. (9). To do so, the first step consists in performing the changes of variables $z-z_{1}=\zeta$, $z^{\prime}-z=\xi$; one thus gets

$$
\begin{aligned}
I_{1}\left(h ; k, k^{\prime} ; z_{1}\right) & \equiv I_{1}\left(h ; k, k^{\prime}\right)=P V \int_{-\infty}^{+\infty} \int_{-\infty}^{+\infty} \frac{f\left(\frac{\zeta}{h}\right) f\left(\frac{\xi}{h}\right)}{\zeta^{2} \xi^{2}}\left(e^{i k \zeta}-1\right)\left(e^{i k^{\prime} \xi}-1\right) e^{i k^{\prime} \zeta} d \zeta d \xi \\
& =P V \int_{-\infty}^{+\infty} \frac{f\left(\frac{\zeta}{h}\right)}{\zeta^{2}}\left(e^{i\left(k+k^{\prime}\right) \zeta}-e^{i k^{\prime} \zeta}\right) d \zeta \cdot P V \int_{-\infty}^{+\infty} \frac{f\left(\frac{\xi}{h}\right)}{\xi^{2}}\left(e^{i k^{\prime} \xi}-1\right) d \xi .
\end{aligned}
$$


Writing then $e^{i\left(k+k^{\prime}\right) \zeta}-e^{i k^{\prime} \zeta}$ as $e^{i\left(k+k^{\prime}\right) \zeta}-1+1-e^{i k^{\prime} \zeta}$, performing the changes of variables $\zeta / h=u$ and $\xi / h=v$, and using the definition (9) of $X$, one gets Eq. (23) $)_{1}$ of the text.

\section{A.2 Integral $I_{2}$}

This integral is given by

$$
\begin{aligned}
I_{2}\left(h ; k, k^{\prime} ; z_{1}\right)=P V \int_{-\infty}^{+\infty} \int_{-\infty}^{+\infty} \frac{f\left(\frac{z^{\prime}-z_{1}}{h}\right) f\left(\frac{z^{\prime}-z}{h}\right)}{\left(z^{\prime}-z_{1}\right)^{2}\left(z^{\prime}-z\right)^{2}} \times \\
\\
\quad\left[e^{i k\left(z^{\prime}-z_{1}\right)}-1-\frac{e^{i k\left(z-z_{1}\right)}-1}{z-z_{1}}\left(z^{\prime}-z_{1}\right)\right]\left[e^{i k^{\prime}\left(z-z_{1}\right)}-1\right] d z d z^{\prime} .
\end{aligned}
$$

Again, one may express it in terms of the sole function $X$. Indeed, one may first perform the changes of variables $z^{\prime}-z_{1}=\zeta, z-z^{\prime}=\xi$; one gets

$$
\begin{aligned}
I_{2}\left(h ; k, k^{\prime} ; z_{1}\right) \equiv I_{2}\left(h ; k, k^{\prime}\right)=P V & \int_{-\infty}^{+\infty} \int_{-\infty}^{+\infty} \frac{f\left(\frac{\zeta}{h}\right) f\left(\frac{\xi}{h}\right)}{\zeta^{2} \xi^{2}} \\
& \times\left(e^{i k \zeta}-1-\frac{e^{i k(\zeta+\xi)}-1}{\zeta+\xi} \zeta\right)\left(e^{i k^{\prime}(\zeta+\xi)}-1\right) d \zeta d \xi .
\end{aligned}
$$

Now, "symmetrizing" the integrand with respect to the variables of integration $\zeta$ and $\xi$, one gets

$$
\begin{aligned}
I_{2}\left(h ; k, k^{\prime}\right)= & \frac{1}{2} P V \int_{-\infty}^{+\infty} \int_{-\infty}^{+\infty} \frac{f\left(\frac{\zeta}{h}\right) f\left(\frac{\xi}{h}\right)}{\zeta^{2} \xi^{2}}\left(e^{i k \zeta}+e^{i k \xi}-1-e^{i k(\zeta+\xi)}\right)\left(e^{i k^{\prime}(\zeta+\xi)}-1\right) d \zeta d \xi \\
= & \frac{1}{2} P V \int_{-\infty}^{+\infty} \int_{-\infty}^{+\infty} \frac{f\left(\frac{\xi}{h}\right) f\left(\frac{\xi}{h}\right)}{\zeta^{2} \xi^{2}}\left(e^{i k \zeta}-1\right)\left(e^{i k \xi}-1\right)\left(1-e^{i k^{\prime}(\zeta+\xi)}\right) d \zeta d \xi \\
= & \frac{1}{2} P V \int_{-\infty}^{+\infty} \frac{f\left(\frac{\zeta}{h}\right)}{\zeta^{2}}\left(e^{i k \zeta}-1\right) d \zeta \cdot P V \int_{-\infty}^{+\infty} \frac{f\left(\frac{\xi}{h}\right)}{\xi^{2}}\left(e^{i k \xi}-1\right) d \xi \\
& -\frac{1}{2} P V \int_{-\infty}^{+\infty} \frac{f\left(\frac{\zeta}{h}\right)}{\zeta^{2}}\left(e^{i\left(k+k^{\prime}\right) \zeta}-e^{i k^{\prime} \zeta}\right) d \zeta \cdot P V \int_{-\infty}^{+\infty} \frac{f\left(\frac{\xi}{h}\right)}{\xi^{2}}\left(e^{i\left(k+k^{\prime}\right) \xi}-e^{i k^{\prime} \xi}\right) d \xi .
\end{aligned}
$$

Reasoning then like for integral $I_{1}$, one gets Eq. $(23)_{2}$ of the text.

\section{B Appendix: Experimental uncertainties on the toughness contrast}

We detail here the method employed to calculate the uncertainty on the experimental values of the toughness contrast $\epsilon$. This contrast is obtained from a linear fit of the variations of the normalized fracture energy $\overline{G_{\mathrm{c}}} / G_{\mathrm{c}}^{\mathrm{M}}$ with the normalized obstacle width $d / b$ following Eq. (52). Therefore, we calculate first the error on the values of the fracture energies at some fixed obstacle width. The effective toughness $\overline{G_{\mathrm{c}}}$ is obtained from the value of the peeling force $F_{\mathrm{p}}$ averaged over a propagation distance of $20 \mathrm{~mm}$ as illustrated in Fig. 7(a). The standard deviation of the force fluctuations over this distance normalized by the average peeling force provides, through Eq. (51), the relative error 
$\sigma_{\overline{G_{\mathrm{c}}}} \simeq 2.0 \%$ on $\overline{G_{\mathrm{c}}}$. The value of the reference fracture energy $G_{\mathrm{c}}^{\mathrm{M}}$ that corresponds to a neat interface is measured from a large set of independent experiments, leading to an improved accuracy $\sigma_{G_{c}^{\mathrm{M}}} \simeq 1.0 \%$. From these two values, one obtains the relative error $\sigma=\sqrt{\left(\sigma_{\overline{G_{\mathrm{c}}}}\right)^{2}+\left(\sigma_{G_{\mathrm{c}}^{\mathrm{M}}}\right)^{2}} \simeq 2.2 \%$ on the normalized values of fracture energy $\overline{G_{\mathrm{c}}} / G_{\mathrm{c}}^{\mathrm{M}}$, that correspond to absolute errors in the range $0.02 \leq \delta \leq 0.03$.

One can now estimate the uncertainty on the contrast value since the linear regression illustrated on Fig. 7 does not only provide the value of $\epsilon$, but also the absolute error $\delta_{\epsilon}$ on this quantity through the formula (Taylor, 1997)

$$
\delta_{\epsilon}=\delta \sqrt{N / \Delta} \text { where } \Delta=\frac{N \sum_{i=1}^{N} d_{i}^{2}-\left(\sum_{i=1}^{N} d_{i}\right)^{2}}{b^{2}}
$$

where the summation is made on the $N=5$ width values $d_{i}$ considered in our experiments. For the specific gray level $c_{\text {gray }}=0.4$ considered in Fig. 7 , this gives $\delta_{\epsilon}=0.06$, leading to the range of contrast values $\epsilon=0.66 \pm 0.06$ used in the first- and second-order theoretical predictions for comparison with experiments (see Fig. 8(a)). Note that the calculation of the error $\delta_{\epsilon}$ through a more elaborated formula than Eq. (B.1), taking into account variations of the error $\delta_{i}$ from one value of $d_{i}$ to another, gives similar results. Finally, this procedure is applied for various gray levels in order to obtain the uncertainties on the values of $\epsilon\left(c_{\text {gray }}\right)$ as represented in Fig. $7(\mathrm{~b})$ for $0 \leq c_{\text {gray }} \leq 1$. This uncertainty is used in Fig. 8(b) when comparing experimental and theoretically predicted values of the front deformation amplitude. 